\title{
FACE FUNCTORS FOR KLR ALGEBRAS
}

\author{
PETER J. MCNAMARA AND PETER TINGLEY
}

\begin{abstract}
Simple representations of KLR algebras can be used to realize the infinity crystal for the corresponding symmetrizable Kac-Moody algebra. It was recently shown that, in finite and affine types, certain sub-categories of "cuspidal" representations realize crystals for sub-Kac-Moody algebras. Here we put that observation on a firmer categorical footing by exhibiting a corresponding functor between the category of representations of the KLR algebra for the sub-Kac-Moody algebra and the category of cuspidal representations of the original KLR algebra.
\end{abstract}

\section{Contents}

1. Introduction ............................................ 107

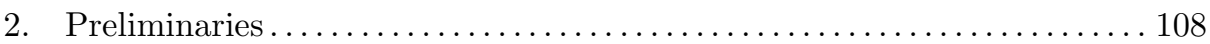

2.1. Root systems and convex orders ......................... 108

2.2. KLR algebras ........................................ 109

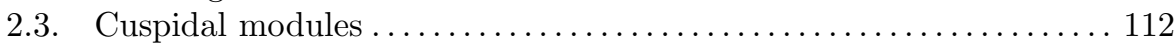

2.4. Crystal structures ................................... 113

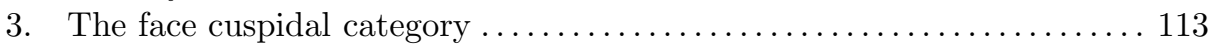

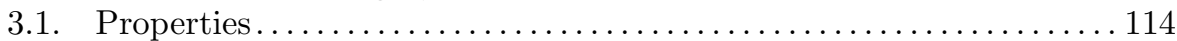

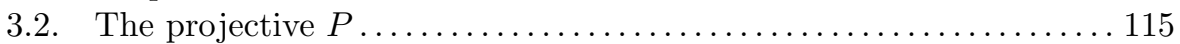

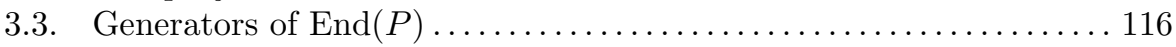

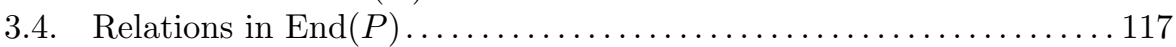

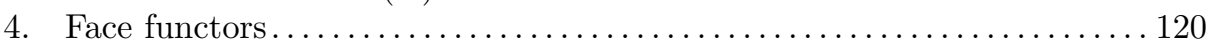

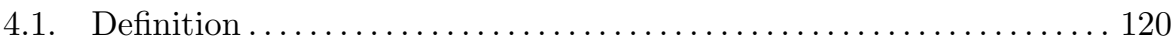

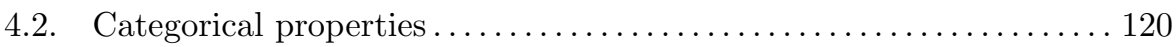

4.3. Compatibility with standard modules......................121

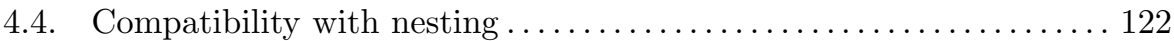

4.5. Compatibility with crystal operators .................... 123

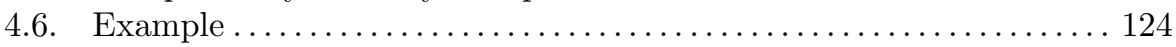

5. Imaginary cuspidal representations and affine faces ............. 124

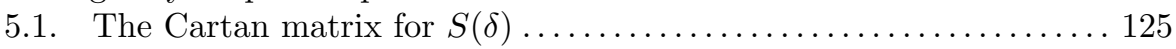

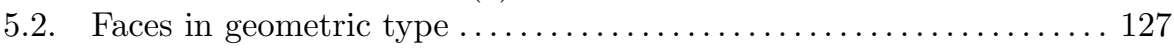

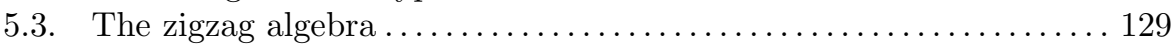

5.4. Example ......................................... 130

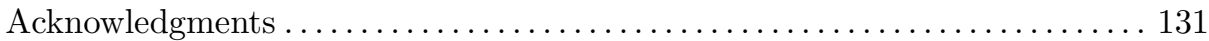

References ................................................ 131

Received by the editors February 12, 2016, and, in revised form, October 3, 2016, March 13, 2017, and May 1, 2017.

2010 Mathematics Subject Classification. Primary 17B37. 


\section{INTRODUCTION}

Khovanov-Lauda-Rouquier (KLR) algebras have been a subject of intense study in the last few years, due to the fact that their categories of graded representations categorify various objects in the theory of quantum groups. Most importantly here, the gradable simple modules for a KLR algebra can be used to realize the crystal $B(-\infty)$ of the positive part of a universal enveloping algebra.

In [TW], the correspondence between simple modules for KLR algebras and crystals is used to define Mirković-Vilonen polytopes in all affine types. There the polytopes arise as the character polytopes of representations of KLR algebras (along with some decoration in affine types). One of the key observations is that, for every face of the MV polytope, there was a sub-category (the cuspidal representations for that face) whose simple representations realized a crystal for a lower rank enveloping algebra. There the correspondence is just combinatorial, but it is natural to ask for a corresponding functor from representations of the smaller rank KLR algebra to representations of the original KLR algebra. Here we construct such a functor under the following conditions:

\section{Assumption 1.1. Either}

- the original root system is of finite type, or

- the original root system is of symmetric affine type, and the KLR algebra is geometric and over a field of characteristic 0 .

In fact, our construction works under somewhat more general conditions, see Assumption 3.11. We construct the functor by considering a certain projective object $P$ in the category of cuspidal representations which has the property that $\operatorname{End}(P)$ is isomorphic to a new KLR algebra. Our face functor is then $X \rightarrow P \otimes_{\operatorname{End}(P)} X$.

If the original KLR algebra satisfies Assumption 1.1 and the face is of finite type, then $P$ is a projective generator and our functor is an equivalence of categories. More generally this is not true, but we still show that the functor respects much of the structure. In particular, it intertwines the crystal operators for the KLR algebra associated to the face and the crystal operators from [TW] for the category of cuspidal modules corresponding to the face whenever both are defined.

We then analyze some implications of our construction for the category of imaginary cuspidal representations of $R(\delta)$ in the affine case. Essentially, our functors allow us to understand all such categories in terms of the $\widehat{\mathfrak{s l}}_{3}$ case. This allows us to show that the category of cuspidal modules of $R(\delta)$ is equivalent to the category of representations of $k[z] \otimes Z$ where $Z$ is the finite type zigzag algebra defined in $\mathrm{HK}$. This last result in the special case of a balanced convex order appears in [KM], where they also consider the cuspidal representations of $R(n \delta)$ for all $n$. Their proof relies on significantly more case-by-case analysis, which our reduction to $\widehat{\mathfrak{s l}}_{3}$ avoids. We believe our arguments should generalize to give their result on cuspidal $R(n \delta)$-modules, which would generalize it to arbitrary convex orders.

Recent work of Kashiwara and Park $[\mathrm{KP}]$ is closely related to our construction. Our face functors are examples of the Kashiwara-Park functors, at least in finite type. Kashiwara and Park do not show that their functors are equivalences of categories for finite type faces (which is not true in the generality they consider), and do not see the connection with the crystal structure in [TW]. 


\section{Preliminaries}

2.1. Root systems and convex orders. Fix a root system $\Phi$. Let $\left(a_{i j}\right)_{i, j \in I}$ be its Cartan matrix, and $d_{i}$ the usual symmetrizing factors. Let $\Delta$ denote the set of simple roots of $\Phi$. For $i, j \in I$, write $i \cdot j$ for $d_{i} a_{i j}$. Then $i \cdot j=j \cdot i$. Extend this by linearity to a $\mathbb{Z}$-valued bilinear pairing on $\mathbb{N} I$. For $\lambda=\left(n_{i}\right)_{i \in I} \in \mathbb{N} I$, define $\operatorname{ht}(\lambda)=\sum_{i \in I} n_{i}$.

Definition 2.1. A face is a decomposition of $\Phi^{+}$into three disjoint subsets

$$
\Phi^{+}=F^{+} \sqcup F \sqcup F^{-}
$$

such that, for all $x \in \operatorname{span}_{\mathbb{R}_{\geq 0}} F$ :

(1) If $y \in \operatorname{span}_{\mathbb{R}_{\geq 0}} F^{+}$is non-zero, then $x+y \notin \operatorname{span}_{\mathbb{R}_{\geq 0}} F^{-} \cup F$.

(2) If $y \in \operatorname{span}_{\mathbb{R}_{\geq 0}} F^{-}$is non-zero, then $x+y \notin \operatorname{span}_{\mathbb{R}_{\geq 0}} F^{+} \cup F$.

We often denote a face simply by $F$, with $F^{+}, F^{-}$being suppressed.

Remark 2.2. The terminology "face" comes from the fact that, in finite type, there is a bijection between faces in the sense of Definition 2.1 and faces of the Weyl polytope (i.e., the convex hull of the Weyl group orbit of $\rho$ ).

Definition 2.3 ([TW, Definition 1.8]). A convex pre-order is a pre-order $\succ$ on $\Phi^{+}$such that, for every equivalence class $C, F^{-}=\{\alpha: \alpha \prec C\}, F=C, F^{+}=\{\alpha$ : $\alpha \succ C\}$ is a face. A convex order is a convex pre-order which is a total order on positive real roots.

Remark 2.4. There is also a notion of convex order based on the condition that if three roots satisfy $\alpha+\beta=\gamma$, then $\gamma$ is between $\alpha$ and $\beta$. It is clear from definitions that any order which is convex according to Definition 2.3 is also convex in that sense. In finite type the two notions were shown to be equivalent in TW, Paragraph before Proposition 1.16]. In affine type the equivalence is shown in [M, Theorem $3.2]$.

Definition 2.5. A convex order $\prec$ is said to be compatible with a face $F$ if $F$ is an interval for $\prec$ and $F^{-} \prec F \prec F^{+}$.

For any linear functional $c: \mathbb{R} \Phi \rightarrow \mathbb{R}$, we get a convex pre-order on $\Phi^{+}$by setting $\alpha \preceq \beta$ if $c(\alpha) / \mathrm{ht}(\alpha) \leq c(\beta) / \mathrm{ht}(\beta)$. The following should be considered a partial converse to this statement.

Lemma 2.6 ([TW, Lemma 1.10]). For any face $F$ there is a sequence $\left\{H_{n}\right\}_{n \in \mathbb{N}}$ of co-oriented hyperplanes in $\mathbb{R} \Phi^{+}$such that

- $F \subset H_{n}$ for all $n$,

- for all $\alpha \in F^{+}, \alpha$ lies on the positive side of $H_{n}$ for $n \gg 0$, and

- for all $\alpha \in F^{-}, \alpha$ lies on the negative side of $H_{n}$ for $n \gg 0$.

In particular, for any $N$, if we let $\Gamma$ be the set of roots in $\Phi^{+}$of height at most $N$, there is a linear functional $c: \mathbb{R} \Phi^{+} \longrightarrow \mathbb{R}$ such that

$$
\left(F^{+} \cap \Gamma, F \cap \Gamma, F^{-} \cap \Gamma\right)=\left(c^{-1}\left(\mathbb{R}_{>0}\right) \cap \Gamma, c^{-1}(0) \cap \Gamma, c^{-1}\left(\mathbb{R}_{<0}\right) \cap \Gamma\right) .
$$

Let $\Delta_{F}$ be the set of positive real roots in $F$ which cannot be written as sums of other positive roots in $F$. Let $\Phi_{F}$ be the corresponding root system whose simple roots are $\Delta_{F}$. If $\Phi$ is at worst affine, then, as discussed in [TW, §3.2], $\Phi_{F}$ is a product of finite and affine root systems (although imaginary root spaces may decompose). 
Remark 2.7. The parenthetical remark above is because, if $\Phi_{F}$ has two or more affine components, it contains non-parallel imaginary roots, all of which come from the imaginary root space of $\Phi$. See [TW, Remark 3.16] for an example.

Lemma 2.8. For every face $F$ there is a compatible convex order $\prec$. Furthermore, for any convex order $\prec_{F}$ on $\Phi_{F}^{+}$, we can choose $\prec$ such that its restriction to $\Phi_{F}^{+}$ agrees with $\prec_{F}$.

Proof. Choose a sequence of linear functionals $\Gamma_{n}$ which define the co-oriented hyperplanes from Lemma 2.6. Define $\alpha \leq \beta$ if $\Gamma_{n}(\alpha) / \mathrm{ht}(\alpha) \leq \Gamma_{n}(\beta) / \mathrm{ht}(\beta)$ for all sufficiently large $n$. This is manifestly a convex pre-order, and $\Phi_{F}^{+}$is an equivalence class. By [TW, Lemma 1.14], this can be refined to a convex total order, which can be made to agree with any chosen convex order on $\Phi_{F}^{+}$.

2.2. KLR algebras. Here we review the Khovanov-Lauda-Rouquier algebras introduced in $\mathrm{KL}, \mathrm{R}]$. For any $\nu \in \mathbb{N} I$, define

$$
\operatorname{Seq}(\nu)=\left\{\mathbf{i}=\left(i_{1}, \ldots, i_{\mathrm{ht}(\nu)}\right) \in I^{\operatorname{ht}(\nu)} \mid \sum_{j=1}^{\operatorname{ht}(\nu)} i_{j}=\nu\right\} .
$$

The symmetric group $S_{\mathrm{ht}(\nu)}$ acts on $\operatorname{Seq}(\nu)$. Let $s_{i}$ denote the adjacent transposition $(i, i+1)$. Fix a field $k$. For each $i, j \in I$, choose $Q_{i j}(u, v) \in k[u, v]$ such that

(Q1) $Q_{i i}(u, v)=0$.

(Q2) If $u$ has degree $2 d_{i}$ and $v$ has degree $2 d_{j}$ then $Q_{i j}$ is homogeneous of degree $-2 d_{i} a_{i j}=-2 d_{j} a_{j i}$. Furthermore, the coefficients $t_{i j}$ and $t_{j i}$ of $u^{-a_{i j}}$ and $v^{-a_{j i}}$ are both non-zero.

(Q3) $Q_{i j}(u, v)=Q_{j i}(v, u)$.

Definition 2.9. The KLR algebra $R(\nu)$ is the associative $k$-algebra generated by elements $e_{\mathbf{i}}, y_{j}, \psi_{k}$ with $\mathbf{i} \in \operatorname{Seq}(\nu), 1 \leq j \leq \operatorname{ht}(\nu)$ and $1 \leq k<\operatorname{ht}(\nu)$, subject to the relations

$$
\begin{aligned}
& e_{\mathbf{i}} e_{\mathbf{j}}=\delta_{\mathbf{i}, \mathbf{j}} e_{\mathbf{i}}, \quad \sum_{\mathbf{i} \in \operatorname{Seq}(\nu)} e_{\mathbf{i}}=1, \quad y_{k} y_{l}=y_{l} y_{k}, \quad y_{k} e_{\mathbf{i}}=e_{\mathbf{i}} y_{k}, \\
& \psi_{l} e_{\mathbf{i}}=e_{s_{l} \mathbf{i}} \psi_{l}, \quad \psi_{k} \psi_{l}=\psi_{l} \psi_{k} \text { if }|k-l|>1, \quad \psi_{k}^{2} e_{\mathbf{i}}=Q_{i_{k}, i_{k+1}}\left(y_{k}, y_{k+1}\right) e_{\mathbf{i}}, \\
& \left(\psi_{k} y_{l}-y_{s_{k}(l)} \psi_{k}\right) e_{\mathbf{i}}= \begin{cases}-e_{\mathbf{i}} & \text { if } l=k, i_{k}=i_{k+1}, \\
e_{\mathbf{i}} & \text { if } l=k+1, i_{k}=i_{k+1}, \\
0 & \text { otherwise, }\end{cases} \\
& \left(\psi_{k+1} \psi_{k} \psi_{k+1}-\psi_{k} \psi_{k+1} \psi_{k}\right) e_{\mathbf{i}} \\
& = \begin{cases}\frac{Q_{i_{k}, i_{k+1}}\left(y_{k}, y_{k+1}\right)-Q_{i_{k}, i_{k+1}}\left(y_{k+2}, y_{k+1}\right)}{y_{k}-y_{k+2}} e_{\mathbf{i}} & \text { if } i_{k}=i_{k+2}, \\
0 & \text { otherwise. }\end{cases}
\end{aligned}
$$

Define $R=\bigoplus_{\nu \in \mathbb{N} I} R(\nu)$.

This is a graded algebra where each $e_{\mathbf{i}}$ has degree zero, $y_{j} e_{\mathbf{i}}$ has degree $i_{j} \cdot i_{j}$ and $\psi_{k} e_{\mathbf{i}}$ has degree $-i_{k} \cdot i_{k+1}$. Fix $\nu$. For each $\sigma \in S_{\mathrm{ht}(\nu)}$, choose a reduced expression 
$\sigma=s_{i_{1}} \cdots s_{i_{k}}$, and let $\psi_{\sigma}=\psi_{i_{1}} \cdots \psi_{i_{k}}$ As shown in [KL, 2.5],

$$
\left\{\psi_{\sigma}\left(\prod_{k=1}^{\mathrm{ht}(\nu)} y_{k}^{r_{k}}\right) e_{\mathbf{i}} \mid \mathbf{i} \in \operatorname{Seq}(\nu), r_{1}, \ldots, r_{\mathrm{ht}(\nu)} \geq 0, \sigma \in S_{\mathrm{ht}(\nu)}\right\}
$$

is a basis for $R(\nu)$.

Throughout this paper we assume all modules are graded and finitely generated. We write $q$ for the grading shift functor, if $V=\bigoplus_{n \in \mathbb{Z}} V_{n}$, then $(q V)_{n}=V_{n-1}$. For modules $U$ and $V$, the space of homomorphisms $\operatorname{Hom}(U, V)$ is graded, we have $\operatorname{Hom}(U, V)=\bigoplus_{n \in \mathbb{Z}} \operatorname{Hom}(U, V)_{n}$, where $\operatorname{Hom}(U, V)_{n}$ is the space of homogenous homomorphisms from $q^{n} U$ to $V$, or equivalently from $U$ to $q^{-n} V$. For a graded vector space $V=\bigoplus_{n \in \mathbb{Z}} V_{n}$, its graded dimension is $\operatorname{dim}_{q} V=\sum_{n \in \mathbb{Z}}\left(\operatorname{dim} V_{n}\right) q^{n}$. If $M$ is an $R(\nu)$ module we say $\operatorname{wt}(M)=\nu$, and define its character to be the formal sum

$$
\operatorname{ch}(M)=\sum_{\mathbf{i}} \operatorname{dim}_{q}\left(e_{\mathbf{i}} M\right)[\mathbf{i}] .
$$

Since we only consider finitely generated modules these dimensions are all finite.

Remark 2.10. There is also a diagrammatic approach to $R$; see [KL, KL2, TW]. There the $y_{i}$ are represented by dots and the $\psi_{i}$ by crossings.

Fix $\lambda, \mu \in \mathbb{N} I$. There is a natural inclusion $\iota_{\lambda, \mu}: R(\lambda) \otimes R(\mu) \rightarrow R(\lambda+\mu)$ defined by $\iota_{\lambda, \mu}\left(e_{\mathbf{i}} \otimes e_{\mathbf{j}}\right)=e_{\mathbf{i j}}, \iota_{\lambda, \mu}\left(y_{i} \otimes 1\right)=y_{i}, \iota_{\lambda, \mu}\left(1 \otimes y_{i}\right)=y_{i+\mathrm{ht}(\lambda)}, \iota_{\lambda, \mu}\left(\psi_{i} \otimes 1\right)=\psi_{i}$, $\iota_{\lambda, \mu}\left(1 \otimes \psi_{i}\right)=\psi_{i+\mathrm{ht}(\lambda)}$. These combine to give an inclusion $\iota: R \otimes R \rightarrow R$. Let $e_{\lambda \mu}$ be the image of the unit under the inclusion $R(\lambda) \otimes R(\mu) \rightarrow R(\lambda+\mu)$.

Definition 2.11. The induction functor $\operatorname{Ind}_{\lambda, \mu}: R(\lambda) \otimes R(\mu)-\bmod \rightarrow R(\lambda+\mu)$-mod is given by

$$
\operatorname{Ind}_{\lambda, \mu}(M)=R(\lambda+\mu) e_{\lambda \mu} \bigotimes_{R(\lambda) \otimes R(\mu)} M
$$

For an $R(\lambda)$-module $A$ and an $R(\mu)$-module $B$, we write $A \circ B$ for $\operatorname{Ind}_{\lambda, \mu}(A \otimes B)$.

The restriction functor $\operatorname{Res}_{\lambda, \mu}: R(\lambda+\mu)$-mod $\rightarrow R(\lambda) \otimes R(\mu)-\bmod$ is given by

$$
\operatorname{Res}_{\lambda, \mu}(M)=e_{\lambda \mu} M \text {. }
$$

By iterating Definition 2.11, functors such as

$$
\begin{aligned}
& \operatorname{Res}_{\lambda_{1}, \ldots, \lambda_{l}}: R\left(\lambda_{1}, \ldots, \lambda_{l}\right)-\bmod \rightarrow R\left(\lambda_{1}\right) \otimes \cdots \otimes R\left(\lambda_{l}\right)-\bmod \\
& \operatorname{Ind}_{\lambda_{1}, \ldots, \lambda_{k}}: R\left(\lambda_{1}\right) \otimes \cdots \otimes R\left(\lambda_{l}\right)-\bmod \rightarrow R\left(\lambda_{1}, \ldots, \lambda_{l}\right)-\bmod
\end{aligned}
$$

can be unambiguously defined.

The induction functor is left adjoint to the restriction functor. Since $\operatorname{Ind}_{\lambda, \mu}$ sends projective modules to projective modules, there is a natural isomorphism

$$
\operatorname{Ext}^{i}\left(M_{1} \circ \cdots \circ M_{n}, N\right) \cong \operatorname{Ext}^{i}\left(M_{1} \otimes \cdots \otimes M_{n}, \operatorname{Res} N\right) \text {. }
$$

The restriction functor also has a right adjoint, given by the usual coinduction construction. We will need [LV], Theorem 2.2], which says

$$
\operatorname{CoInd}(A \otimes B) \cong q^{-\lambda \cdot \mu} B \circ A .
$$

In [LV] this is stated for finite dimensional modules, but the same proof shows it is true in complete generality. Thus, if $A$ is an $R(\lambda+\mu)$-module, $B$ is an $R(\lambda)$-module and $C$ is an $R(\mu)$-module, then there is a natural isomorphism

$$
\operatorname{Hom}(\operatorname{Res} A, B \otimes C) \cong \operatorname{Hom}\left(A, q^{-\lambda \cdot \mu} C \circ B\right) \text {. }
$$


The following is often called the Mackey filtration.

Theorem 2.12 ([ $\left[\mathrm{KL}\right.$, Proposition 2.18]). Let $\lambda_{1}, \ldots, \lambda_{k}, \mu_{1} \ldots, \mu_{l} \in \mathbb{N} I$ be such that $\sum_{i} \lambda_{i}=\sum_{j} \mu_{j}$, and let $M$ be an $R\left(\lambda_{1}\right) \otimes \cdots \otimes R\left(\lambda_{k}\right)$-module. Then the composition

$$
\operatorname{Res}_{\mu_{1}, \ldots, \mu_{l}} \circ \operatorname{Ind}_{\lambda_{1}, \ldots, \lambda_{k}}(M)
$$

has a filtration indexed by tuples $\eta_{i j}$ satisfying $\lambda_{i}=\sum_{j} \eta_{i j}$ and $\mu_{j}=\sum_{i} \eta_{i j}$. Every sub-quotient of this filtration is isomorphic to $\operatorname{Ind}_{\eta}^{\mu} \circ \tau \circ \operatorname{Res}_{\eta}^{\lambda}(M)$, where

- $\operatorname{Res}_{\eta}^{\lambda}: \otimes_{i} R\left(\lambda_{i}\right)$-mod $\longrightarrow \otimes_{i}\left(\otimes_{j} R\left(\eta_{i j}\right)\right)$-mod is the tensor product of the $\operatorname{Res}_{\eta_{i \bullet}}$

- $\tau: \otimes_{i}\left(\otimes_{j} R\left(\eta_{i j}\right)\right)$-mod $\longrightarrow \otimes_{j}\left(\otimes_{i} R\left(\eta_{i j}\right)\right)$-mod is given by permuting the tensor factors and shifting the degree. The degree shift is the degree of the permutation acting on the idempotent $e_{\eta_{11} \eta_{12} \cdots \eta_{k \ell}}$, and

- $\operatorname{Ind}_{\eta}^{\mu}: \otimes_{j}\left(\otimes_{i} R\left(\eta_{i j}\right)\right)-\bmod \longrightarrow \otimes_{j} R\left(\mu_{j}\right)$-mod is the tensor product of the $\operatorname{Ind}_{\eta_{\bullet}:}$.

There is an anti-automorphism $\dagger$ of $R$ which fixes each of the generators $e_{\mathbf{i}}, y_{j}$ and $\psi_{k}$. Thus, given a finite dimensional $R$-module $M$, we can define the structure of an $R$-module on its dual $M^{\circledast}=\operatorname{Hom}_{k}(M, k)$ by $(r \cdot \phi)(m)=\phi\left(r^{\dagger} \cdot m\right)$. As in [KL, §3.2] each finite dimensional simple module is isomorphic to its dual up to a grading shift. The dual induces the bar involution on the Grothendieck group. Its behavior with respect to induction is

$$
(M \circ N)^{\circledast} \cong q^{\mathrm{wt}(M) \cdot \mathrm{wt}(N)} N^{\circledast} \circ M^{\circledast} .
$$

As discussed in KL2, different choices of $Q_{i j}$ can produce isomorphic algebras.

Definition 2.13. Let $R$ be a KLR algebra of symmetric type. We say $R$ is of geometric type if $R$ is isomorphic to the one defined in [M] via an isomorphism $\phi$ which respects the inclusion $\iota: R \otimes R \rightarrow R$ in the sense that $\phi \circ \iota=\iota \circ(\phi \otimes \phi)$.

The terminology is due to a geometric interpretation of KLR algebras with this choice of parameters. The following is discussed by Khovanov and Lauda [KL2].

\section{Lemma 2.14.}

(1) Every KLR algebra whose Dynkin diagram is a tree is of geometric type. In particular, every KLR algebra of affine type $D_{n}$ and $E_{n}$ is of geometric type.

(2) For $n \geq 3$, a KLR algebra of type $\widehat{\mathfrak{s l}}_{n}$ with $I=\mathbb{Z} / n \mathbb{Z}$ and $Q_{i, i+1}=s_{i} u+t_{i} v$ is of geometric type if and only if $s_{1} \cdots s_{n} / t_{1} \cdots t_{n}=(-1)^{n}$.

(3) A KLR algebra of type $\widehat{\mathfrak{s l}}_{2}$ is of geometric type if and only if the quadratic polynomial $Q_{0,1}(u, v)$ has discriminant zero.

Proof. Fix a root system. There is an action of $\left(k^{*}\right)^{I \times I}$ on the set of KLR algebras where $\left(z_{i j}\right)_{i, j \in I}$ acts by $\psi_{j} e_{\mathbf{i}} \mapsto z_{i_{j} i_{j+1}} \psi_{j} e_{\mathbf{i}}, y_{j} e_{\mathbf{i}} \mapsto z_{i_{j} i_{j}}^{-1} y_{j} e_{\mathbf{i}}$ and $e_{\mathbf{i}} \mapsto e_{\mathbf{i}}$. This sends algebras to isomorphic algebras. The $Q_{i j}$ change according to $Q_{i j}^{\prime}(u, v)=$ $z_{i j} z_{j i} Q_{i j}\left(z_{i i} u, z_{j j} v\right)$. Furthermore, all possible isomorphisms of KLR algebras that fix the idempotents $e_{\mathbf{i}}$ arise in this way. To see this notice that, for $i \neq j$, looking at only 2 strands, it is clear for weight reasons that any automorphism must send $\psi_{1} e_{\mathbf{i}}$ to a multiple of itself, say $z_{i_{1} i_{2}} \psi_{1} e_{\mathbf{i}}$. Also, looking at a single strand, $y_{1}$ must be sent to a multiple of itself, say $z_{i_{1} i_{1}} y_{1}$. The relation that, if $i_{1}=i_{2},\left(\psi_{1} y_{2}-y_{1} \psi_{1}\right) e_{\mathbf{i}}=e_{\mathbf{i}}$ implies that a crossing with both stands colored $i$ must be scaled by $z_{i i}^{-1}$. 
If the Dynkin diagram is a tree, it is clear that all choices of $Q_{i j}$ are related by such isomorphisms. This establishes (1).

In type $\widehat{\mathfrak{s l}}_{n}$, for the KLR algebras used in [M], it is easy to check that the stated relations hold. The action of $\left(k^{*}\right)^{I \times I}$ preserves these relations, as do diagram automorphisms, so they hold for all geometric type KLR algebras. Furthermore, one can check that all KLR algebras that satisfy these conditions are related in this way, establishing (2) and (3).

Definition 2.15. A pseudo-KLR algebra is a KLR algebra in the sense of Definition 2.9 with the requirement that $t_{i j} \neq 0$ in Condition (Q2) removed.

The following demonstrates that pseudo-KLR algebras which are not in fact KLR algebras have noticeably different categories of representations. Fix $i \neq j$. Due to the categorification results from [KL], for an actual KLR algebra $R\left(\left(1-a_{i j}\right) i+j\right)$, the number of irreducible modules up to grading shift is exactly the dimension of that weight space of $U^{+}$, so $1-a_{i j}$.

Theorem 2.16. In a pseudo-KLR algebra with the coefficient $t_{i j}=0$ for some $i \neq j$, the algebra $R\left(\left(1-a_{i j}\right) i+j\right)$ has at least $2-a_{i j}$ irreducible modules.

Proof. For each $a, b \in \mathbb{Z}_{\geq 0}$ with $a+b=1-a_{i j}$, consider the irreducible $R(a i) \otimes$ $R(j) \otimes R(b i)$-module $L_{a, b}=L\left(i^{a}\right) \otimes L(j) \otimes L\left(i^{b}\right)$. We claim that $L_{a, b}$ is in fact a module for $R\left(\left(1-a_{i j}\right) i+j\right)$, where all generators which are not in $R(a i) \otimes R(j) \otimes R(b i)$ act by zero. To see this, one must check that the relations defining the KLR algebra are all respected. This happens because, since $t_{i j}=0$, the polynomial $Q_{i j}(u, v)$ is divisible by $v$, which corresponds to a dot $y_{a+1}$ on the $j$ colored strand, and this dot kills the simple $L(j)$. The right side of the difficult relations (the ones involving multiple $\psi$ 's and which involve the $j$ strand) then all have a factor of $y_{a+1}$.

Each $L_{a, b}$ is irreducible since its restriction to $R(a i) \otimes R(j) \otimes R(b i)$ is irreducible. Thus we have found $2-a_{i j}$ distinct irreducible modules.

2.3. Cuspidal modules. For the remainder of this section, fix a face $F$ and a compatible convex order $\prec$. An $R$-module $M$ is called $F$-cuspidal if, for all $\lambda, \mu \in \mathbb{N} I$ such that $\operatorname{Res}_{\lambda, \mu} M \neq 0$,

$$
\lambda \in \operatorname{span}_{\mathbb{R}_{\geq 0}}\left(F^{-} \cup F\right) \quad \text { and } \quad \mu \in \operatorname{span}_{\mathbb{R}_{\geq 0}}\left(F^{+} \cup F\right) .
$$

Fix a module $M$ and $c$ as in Lemma 2.6 for $N=\operatorname{ht}(\operatorname{wt}(M))$. In [TW], $M$ is called semi-cuspidal if $\operatorname{Res}_{\lambda, \mu} M \neq 0$ implies $c(\lambda) \leq 0$ (in that paper there is a stronger notion of cuspidal, which is why the term semi-cuspidal is used). This notion of semi-cuspidal is equivalent to our notion of cuspidal, but to see this one must use [TW, Theorem 2.4 and Corollary 2.12], which imply that, if $\operatorname{Res}_{\lambda, \mu} M \neq 0$ and $c(\lambda)>0$, then there is a root $\beta$ with $\operatorname{Res}_{\beta, w t(\lambda)-\beta} M \neq 0$ and $c(\beta)>0$. In any case, we see:

Lemma 2.17. Fix a module $M$ and $c$ as in Lemma 2.6 such that, for roots $\beta$ of height at most $\operatorname{wt}(M), \beta$ is in $F^{+} / F / F^{-}$if and only if $c(\beta)$ is positive/zero/negative. Then $M$ is cuspidal if and only if $\operatorname{Res}_{\lambda, \mu} M \neq 0$ implies $c(\lambda) \leq 0$.

Definition 2.18. Let $\mathcal{C}_{F}$ denote the full sub-category of cuspidal $R$-modules.

Recall from Definition 2.3 that each equivalence class for a convex pre-order $\succ$ is a face. We call a module $\succ$-cuspidal if it is cuspidal for some equivalence class. 
Theorem 2.19 ([TW, Theorem 2.19]). If $L_{1}, \ldots, L_{h}$ are simple, $\succ$-cuspidal, and satisfy $\operatorname{wt}\left(L_{1}\right) \succ \cdots \succ \operatorname{wt}\left(L_{h}\right)$, then $L_{1} \circ \cdots \circ L_{h}$ has a unique simple quotient. Furthermore, every simple appears this way up to a grading shift for a unique sequence of simple cuspidal representations. Here $\operatorname{wt}\left(L_{i}\right) \succ \operatorname{wt}\left(L_{j}\right)$ means that these are in the positive spans of some equivalence classes $C_{i}, C_{j}$, and $C_{i} \succ C_{j}$.

Corollary 2.20 ([TW, Corollary 2.17]). Fix a convex total order $\succ$ and a positive real root $\alpha$. There is a unique self-dual simple cuspidal $R(\alpha)$-module $L(\alpha)$.

Definition 2.21. Let $\alpha$ be a positive real root. Let $F(\alpha)$ be the face defined by

$$
F^{+}=\{\beta \mid \beta \succ \alpha\}, \quad F=\{\alpha\}, \quad F^{-}=\{\beta \mid \beta \prec \alpha\} .
$$

Define the root module $\Delta(\alpha)$ to be the projective cover of $L(\alpha)$ in $\mathcal{C}_{F(\alpha)}$.

\subsection{Crystal structures.}

Definition 2.22. Let $\mathcal{B}$ be the set of simple self-dual elements of $R$-mod. For each face $F$ let $\underline{\mathcal{B}}_{F}$ be the set of self-dual simple elements of the cuspidal category $\mathcal{C}_{F}$.

Theorem 2.23 ([LV] $) \cdot \mathcal{B}$ is isomorphic to a copy of $B(\infty)$ for the root system $\Phi$, where the crystal operators are given by $f_{i}(L)=q_{i}^{\epsilon_{i}(L)} \mathrm{hd}(L \circ L(i))$.

Remark 2.24. The precise grading shift is not given in [LV]. It can be found without proof in [KKKO, Definition 4.16(iii)]. For completeness here is a proof: By the definition of $\epsilon_{i}$,

$$
\operatorname{Res}_{\mathrm{wt}(L)-\epsilon_{i}(L) i, \epsilon_{i}(L) i} L \simeq L^{\prime} \otimes L\left(\epsilon_{i}(L) i\right) \text { and } \operatorname{Res}_{\mathrm{wt}(L)-\left(\epsilon_{i}(L)+1\right) i,\left(\epsilon_{i}(L)+1\right) i} L=0
$$

for a self-dual $L^{\prime}$. By rank 1 calculations, $L\left(\epsilon_{i}(L) i\right) \circ L(i)=q_{i}^{-\epsilon_{i}(L)} L\left(\left(\epsilon_{i}(L)+1\right) i\right)$. It follows that

$$
\operatorname{Res}_{\mathrm{wt}(L)-\epsilon_{i}(L) i,\left(\epsilon_{i}(L)+1\right) i,} L \circ L(i) \simeq q_{i}^{-\epsilon_{i}(L)} L^{\prime} \otimes L\left(\left(\epsilon_{i}(L)+1\right) i\right) .
$$

Since the restriction of a self-dual representation is self-dual the statement follows.

Theorem 2.25 ([TW]). Assume $\Phi$ is at worst affine. Then:

- If $F$ is finite type, $\underline{\mathcal{B}}_{F}$ is isomorphic to a copy of $B(\infty)$ for the root system $\Phi_{F}$. The crystal operators are given by, for each $\underline{i} \in \Delta_{F}, f_{\underline{i}}(L)=$ $q_{i}^{\epsilon_{i}}(L) \operatorname{hd}(L \circ L(\underline{i}))$.

- If $\Phi_{F}$ has one or more affine components, $\underline{\mathcal{B}}_{F}$ is isomorphic to a union of infinitely many copies of $B(\infty)$ for $\Phi_{F}$, with crystal operators $f_{\underline{i}}(L)=$ $q_{\underline{\underline{i}}}^{\epsilon_{\underline{i}}(L)} \operatorname{hd}(L \circ L(\underline{i}))$.

Remark 2.26. The grading shift is not specified in [TW, but follows by an argument similar to Remark 2.24. The key step is that, for some self-dual $L^{\prime}$,

$$
\left.\operatorname{Res}_{R\left(\operatorname{wt}(L)-\epsilon_{\underline{i}}(L) \underline{i}\right), R(\underline{i})}\right)^{\otimes \epsilon_{\underline{i}}(L)+1} L \circ L(\underline{i}) \simeq q_{\underline{i}}^{-\epsilon_{\underline{i}}(L)}\left[\epsilon_{\underline{i}}(L)+1\right] ! L^{\prime} \otimes L(\underline{i})^{\otimes \epsilon_{\underline{i}}(L)+1} .
$$

\section{The FACE Cuspidal CATEgory}

Fix a root system $\Phi$ and let $R$ be an associated KLR algebra. Fix a face $F$ and a compatible convex order $\prec$ (which is possible by Lemma 2.8). Let $\mathcal{C}$ be the full sub-category of $R$-mod consisting of $F$-cuspidal representations (which is $\mathcal{C}_{F}$ from 2.3). The main purpose of this section is to define a projective object $P$ in $\mathcal{C}$, and, under certain conditions, show that $\operatorname{End}(P)$ is isomorphic to a KLR algebra for the root system $\Phi_{F}$. 


\subsection{Properties.}

Lemma 3.1. $\mathcal{C}$ is abelian and is closed under $\circ$. That is, if $M, N \in \mathcal{C}$, then $M \circ N \in \mathcal{C}$.

Proof. It is clear that $\mathcal{C}$ is abelian. Fix $M, N$ and choose $c$ as in $\$ 2.3$ for wt $(M)+$ $\operatorname{wt}(N)$. By the definition of $\circ$, if $e_{\mathbf{i}}(M \circ N) \neq 0$, then $\mathbf{i}$ is a shuffle of some $\mathbf{i}_{1}$ and $\mathbf{i}_{2}$ such that $e_{\mathbf{i}_{1}} M \neq 0$ and $e_{\mathbf{i}_{2}} N \neq 0$. Thus, if $\mathbf{i}^{\prime}$ is a prefix of $\mathbf{i}$, then $\mathbf{i}^{\prime}$ is a shuffle of a prefix $\mathbf{i}_{1}^{\prime}$ of $\mathbf{i}_{1}$ and $\mathbf{i}_{2}^{\prime}$ of $\mathbf{i}_{2}$. But then $c\left(\mathbf{i}_{1}^{\prime}\right), c\left(\mathbf{i}_{2}^{\prime}\right) \leq 0$, so $c\left(\mathbf{i}^{\prime}\right) \leq 0$. This holds for all $\mathbf{i}$ such that $e_{\mathbf{i}}(M \circ N) \neq 0$ and all prefixes, so $M \circ N \in \mathcal{C}$ by definition.

Lemma 3.2. For any $\alpha \in \Delta_{F}$ the modules $L(\alpha)$ and $\Delta(\alpha)$ depend only on the face, not on the choice of compatible convex order.

Proof. Fix convex orders $\prec, \prec^{\prime}$ compatible with $F$. Let $L(\alpha)$ be the simple cuspidal for $\alpha$ with respect to $\prec$. Consider the cuspidal decomposition

$$
L(\alpha)=\operatorname{hd}\left(L_{1}^{\prime} \circ \cdots \circ L_{k}^{\prime}\right)
$$

from Theorem 2.19 with respect to $\prec^{\prime}$. If $\operatorname{wt}\left(L_{1}^{\prime}\right)$ is not in $F$, then

$$
\operatorname{Res}_{\mathrm{wt}\left(L_{1}^{\prime}\right), \alpha-\mathrm{wt}\left(L_{1}^{\prime}\right)} L(\alpha) \neq 0
$$

contradicts the cuspidality of $L(\alpha)$ with respect to $\prec$, and similarly for $L_{n}^{\prime}$. Thus, for all $k, \operatorname{wt}\left(L_{k}^{\prime}\right)=s_{k} \beta_{k}$ for $\beta_{k} \in F$. But $\alpha=\sum s_{k} \beta_{k}$ contradicts $\alpha \in \Delta_{F}$ unless the cuspidal decomposition is only one step. Thus $L(\alpha)$ is cuspidal for $\prec^{\prime}$. Since $L(\alpha)$ is the unique simple of weight $\alpha$ in $\mathcal{C}$ defined using either $\prec$ or $\prec^{\prime}$, it follows that $\Delta(\alpha)$ also does not depend on this choice.

Lemma 3.3. Let $\beta_{1}, \ldots, \beta_{n}, \gamma_{1}, \ldots, \gamma_{n} \in \Delta_{F}$ with $\sum \beta_{i}=\sum \gamma_{j}$. Let $M_{1}, \ldots, M_{n}$ be face-cuspidal modules with $\operatorname{wt}\left(M_{i}\right)=\beta_{i}$. Consider

$$
\operatorname{Res}_{\gamma_{1}, \ldots, \gamma_{n}}\left(M_{1} \circ \cdots \circ M_{n}\right) \text {. }
$$

The non-zero layers in the Mackey filtration from Theorem 2.12 are exactly those corresponding to permutations of the factors (i.e., where for some permutation $\sigma$, $\eta_{i j}=0$ unless $\left.j=\sigma(i)\right)$. The sub-quotients are of the form $M_{\sigma^{-1}(1)} \otimes \cdots \otimes M_{\sigma^{-1}(n)}$, with a shift by the degree of $\sigma$ acting on the idempotent $e_{\beta_{1} \cdots \beta_{n}}$. In particular, the restriction is non-zero if and only if $\left\{\beta_{1}, \ldots, \beta_{n}\right\}=\left\{\gamma_{1}, \ldots, \gamma_{n}\right\}$ as multisets.

Proof. It is clear that there is a sub-quotient $\operatorname{Res}_{\gamma_{1}, \ldots, \gamma_{n}}\left(M_{1} \circ \cdots \circ M_{n}\right)$ in the Mackey filtration corresponding to any permutation $\sigma \in S_{n}$ such that $\gamma_{\sigma(i)}=\beta_{i}$ for all $i$. Suppose there is a non-zero sub-quotient that does not correspond to such a permutation. Let $k$ be the first index such that $\gamma_{k}$ is a non-trivial sum $\gamma_{k}=\sum_{i} \eta_{i k}$. For each $i$ such that $\eta_{i k} \neq 0$, the minimality of $k$ implies that $\operatorname{Res}_{\eta_{i k}, \beta_{i}-\eta_{i k}} M_{i} \neq 0$. The face-cuspidality of $M_{i}$ implies that $c\left(\eta_{i k}\right) \leq 0$, where $c$ is a functional compatible with $F$ as in Lemma 2.6 for $N=\sum \beta_{i}$. Since $\gamma_{k}$ is a simple root in the face $F, \eta_{i k}$ does not lie in $F$ and hence, $\beta_{i}, c\left(\eta_{i k}\right)<0$. Since $\gamma_{k}=\sum_{i} \eta_{i k}$, convexity implies that $c\left(\gamma_{k}\right)<0$, a contradiction.

Lemma 3.4. Fix $\underline{i}_{1}, \ldots, \underline{i}_{n} \in \Delta_{F}$ and $M \in \mathcal{C}$. Every simple sub-quotient of $\operatorname{Res}_{\underline{i}_{1}, \ldots, \underline{i}_{n}} M$ is of the form $L\left(\underline{i}_{1}\right) \otimes \cdots \otimes L\left(\underline{i}_{n}\right)$ up to a grading shift.

Proof. By Lemma 2.6, we can choose a linear functional $c$ such that $F \subset c^{-1}(0)$ and, for any root $\gamma$ of height at most ht $(\operatorname{wt}(M)), \gamma \in F^{-}$if and only if $c(\beta)<0$, and $\beta \in F^{+}$if and only if $c(\beta)>0$. 
Let $M_{1} \otimes \cdots \otimes M_{n}$, be a simple sub-quotient of $\operatorname{Res}_{\underline{i}_{1}, \ldots, \underline{i}_{n}} M$. Assume that, for some $j, M_{j}$ is not isomorphic to $L\left(\underline{i}_{j}\right)$, and take $j$ minimal for this to occur. Let $M_{j}=\operatorname{hd}\left(N_{1} \circ \ldots \circ N_{k}\right)$ be the decomposition of $M_{j}$ as in Theorem 2.19 for the corresponding convex pre-order, and let $\lambda=\underline{i}_{1}+\cdots+\underline{i}_{j-1}+\operatorname{wt}\left(N_{1}\right)$. Since $\underline{i}_{j}$ is simple for $\Phi_{F}, \operatorname{wt}\left(N_{1}\right)$ cannot be a multiple of a root in $F$, so $c\left(N_{1}\right)>c\left(M_{j}\right)=0$. But then

$$
e_{\lambda, \mathrm{wt}(M)-\lambda} M \neq 0 \quad \text { and } \quad c(\lambda)=0+\cdots+0+c\left(\operatorname{wt}\left(N_{1}\right)\right)>0 .
$$

By Lemma 2.17, this contradicts the assumption that $M$ was cuspidal.

\subsection{The projective $P$.}

Theorem 3.5. Let $\underline{i}_{1}, \ldots, \underline{i}_{n} \in \Delta_{F}$. Then $\Delta\left(\underline{i}_{1}\right) \circ \cdots \circ \Delta\left(\underline{i}_{n}\right)$ is projective in $\mathcal{C}$.

Proof. By Lemma 3.1, $\Delta\left(\underline{i}_{1}\right) \circ \cdots \circ \Delta\left(\underline{i}_{n}\right) \in \mathcal{C}$. To show projectivity, it is equivalent to show that

$$
\operatorname{Ext}^{1}\left(\Delta\left(\underline{i}_{1}\right) \circ \cdots \circ \Delta\left(\underline{i}_{n}\right), M\right)=0
$$

for all $M \in \mathcal{C}$. By the adjunction (2.2), this is equivalent to showing

$$
\operatorname{Ext}^{1}\left(\Delta\left(\underline{i}_{1}\right) \otimes \cdots \otimes \Delta\left(\underline{i}_{n}\right), \operatorname{Res}_{\underline{i}_{1}, \ldots, \underline{i}_{n}} M\right)=0 .
$$

By Lemma 3.4, it suffices to prove

$$
\operatorname{Ext}^{1}\left(\Delta\left(\underline{i}_{1}\right) \otimes \cdots \otimes \Delta\left(\underline{i}_{n}\right), L\left(\underline{i}_{1}\right) \otimes \cdots \otimes L\left(\underline{i}_{n}\right)\right)=0 .
$$

Since $\operatorname{Ext}^{1}\left(\Delta\left(\underline{i}_{k}\right), L\left(\underline{i}_{k}\right)\right)=0$, this is true.

Definition 3.6. Let $P$ be the direct sum of all modules of the form $\Delta\left(\underline{i}_{1}\right) \circ \cdots \circ \Delta\left(\underline{i}_{n}\right)$ for $\underline{i}_{1}, \ldots, \underline{i}_{n} \in \Delta_{F}$. The indexing set for these direct summands is $\coprod_{\nu} \operatorname{Seq}_{F}(\nu)$ where

$$
\operatorname{Seq}_{F}(\nu)=\left\{\left(\underline{i}_{1}, \ldots, \underline{i}_{n}\right): \underline{i}_{k} \in \Delta_{F}, \underline{i}_{1}+\cdots+\underline{i}_{n}=\nu\right\} .
$$

For $\underline{\mathbf{i}}=\left(\underline{i}_{1}, \ldots, \underline{i}_{n}\right) \in \operatorname{Seq}_{F}(\nu)$, we write $\Delta\left(\underline{\mathbf{i}}_{)}\right)$for $\Delta\left(\underline{i}_{1}\right) \circ \cdots \circ \Delta\left(\underline{i}_{n}\right)$.

Theorem 3.7. If $\Phi$ is of finite or affine type and $\Phi_{F}$ is of finite type, then $P$, together with its grading shifts, is a projective generator of $\mathcal{C}$.

Proof. By Theorem 3.5, $\Delta\left(\underline{i}_{1}\right) \circ \cdots \circ \Delta\left(\underline{i}_{n}\right)$ is projective. It suffices to show that every simple module $L$ in $\mathcal{C}$ is a quotient of a module of the form $\Delta\left(\underline{i}_{1}\right) \circ \cdots \circ \Delta\left(\underline{i}_{n}\right)$. By [TW, Corollary 3.29], every non-trivial simple in $\mathcal{C}$ is of the form $\operatorname{hd}(L(\underline{i}) \circ X)$ for some $\underline{i} \in \Delta_{F}$ and some simple $X$ in $\mathcal{C}$, so by induction is a quotient of a module of the form $\Delta\left(\underline{i}_{1}\right) \circ \cdots \circ \Delta\left(\underline{i}_{n}\right)$.

Remark 3.8. A counterexample to Theorem 3.7 when $\Phi$ and $\Phi_{F}$ are both of affine type is given in \$5.4. A counterexample when $\Phi$ is of hyperbolic type and $\Phi_{F}$ is finite type is provided in [TW, §3.7]. There $\Phi_{F}$ is of type $\mathfrak{s l}_{2}$, but there are also imaginary cuspidal modules, contradicting the statement.

Fix $\underline{\mathbf{i}}=\left(\underline{i}_{1}, \cdots, \underline{i}_{n}\right), \underline{\mathbf{j}}=\left(\underline{i}_{1}, \cdots, \underline{i}_{n}\right) \in \operatorname{Seq}_{F}(\nu)$. Let ${ }_{\mathbf{j}} S_{\underline{\mathbf{i}}}$ be the subset of the symmetric group $S_{n}$ consisting of permutations taking $\underline{\mathbf{i}} \underline{\mathbf{j}}$ to $\underline{\mathbf{j}}$. For each $w \in{ }_{\mathbf{j}} S_{\underline{\mathbf{i}}}$, pick a reduced decomposition $w=s_{i_{1}} \cdots s_{i_{n}}$. Set

$$
\tau_{w}(\underline{\mathbf{i}})=\tau_{i_{1}}\left(s_{i_{2}} \cdots s_{i_{n}} \underline{\mathbf{i}}\right) \cdots \tau_{i_{n}}(\underline{\mathbf{i}}) .
$$

Define $\operatorname{deg}_{\mathbf{i}}(w)$ to be $\operatorname{deg} \tau_{w}(\mathbf{i})$, which clearly does not depend on the reduced decomposition. 
Lemma 3.9. Fix $\underline{\mathbf{i}}, \underline{\mathbf{j}} \in \operatorname{Seq}_{F}(\nu)$. Then $\operatorname{Res}_{\underline{\mathbf{j}}} \Delta(\underline{\mathbf{i}}) \cong \bigoplus_{w \in \underline{\mathbf{j}} S_{\underline{\mathbf{i}}}} q^{\operatorname{deg}_{\mathbf{i}}(w)} \Delta\left(j_{1}\right) \otimes \cdots \otimes \Delta\left(j_{n}\right)$.

Proof. By Lemma 3.3 there is a filtration of $\operatorname{Res}_{\mathbf{j}} \Delta(\underline{\mathbf{i}})$ with these sub-quotients. This filtration splits since $\operatorname{Ext}^{1}\left(\Delta\left(j_{i}\right), \Delta\left(j_{i}\right)\right)=0$.

3.3. Generators of $\operatorname{End}(P)$. Write $P=\bigoplus_{\nu} P_{\nu}$ where $P_{\nu}$ is an $R(\nu)$-module. Given a sequence $\underline{\mathbf{i}}=\left(\underline{i}_{1}, \ldots, \underline{i}_{n}\right)$ of elements in $\Delta_{F}$, let

$$
e_{\underline{\mathbf{i}}} \in \operatorname{End}(P)
$$

be the projection onto the summand $\Delta\left(\underline{i}_{1}\right) \circ \cdots \circ \Delta\left(\underline{i}_{n}\right)$.

For $m, n \in \mathbb{N}$, let $w[m, n]$ be the element of the symmetric group $S_{m+n}$ given by

$$
w[m, n](i)= \begin{cases}i+n & \text { if } i \leq m, \\ i-m & \text { otherwise }\end{cases}
$$

There is a unique reduced expression $s_{i_{1}} \cdots s_{i_{l}}$ for $w[m, n]$ up to two-term braid moves (since it has a unique descent). Thus we can unambiguously define an element $\psi_{w[m, n]}=\psi_{i_{1}} \cdots \psi_{i_{l}} \in R$.

For each positive real root $\alpha$, fix a non-zero vector $v_{\alpha} \in \Delta(\alpha)$ of minimal degree.

Lemma 3.10. Fix $\underline{i}, \underline{k} \in \Delta_{F}$. There is a unique homomorphism

$$
\tau: q^{-\underline{i} \cdot \underline{k}} \Delta(\underline{i}) \circ \Delta(\underline{k}) \longrightarrow \Delta(\underline{k}) \circ \Delta(\underline{i})
$$

such that $\tau\left(1 \otimes\left(v_{\underline{i}} \otimes v_{\underline{k}}\right)\right)=\psi_{w[h t(\underline{i}), h t(\underline{k})]} \otimes\left(v_{\underline{k}} \otimes v_{\underline{i}}\right)$.

Proof. If $\underline{i}=\underline{k}$, this is [BKM, Lemma 3.6] (there it is assumed that $\Phi$ is finite type, but the same proof works in general). Now assume that $\underline{i} \neq \underline{k}$. By adjunction,

$\operatorname{Hom}\left(q^{-\underline{i} \cdot \underline{k}} \Delta(\underline{i}) \circ \Delta(\underline{k}), \Delta(\underline{k}) \circ \Delta(\underline{i})\right) \cong \operatorname{Hom}\left(q^{-\underline{i} \cdot \underline{k}} \Delta(\underline{i}) \otimes \Delta(\underline{k}), \operatorname{Res}_{\underline{i}, \underline{k}} \Delta(\underline{k}) \circ \Delta(\underline{i})\right)$.

By Lemma 3.3. the Mackey filtration of $\operatorname{Res}_{\underline{i}, \underline{k}} \Delta(\underline{k}) \circ \Delta(\underline{i})$ from Theorem 2.12 has a unique non-zero layer, resulting in an isomorphism

$$
\operatorname{Res}_{\underline{i}, \underline{k}} \Delta(\underline{k}) \circ \Delta(\underline{i}) \cong q^{-\underline{i} \cdot \underline{k}} \Delta(\underline{i}) \otimes \Delta(\underline{k}) .
$$

Thus

$$
\operatorname{Hom}\left(q^{-\underline{i} \cdot \underline{k}} \Delta(\underline{i}) \circ \Delta(\underline{k}), \Delta(\underline{k}) \circ \Delta(\underline{i})\right) \cong \operatorname{Hom}\left(q^{-\underline{i} \cdot \underline{k}} \Delta(\underline{i}) \otimes \Delta(\underline{k}), q^{-\underline{i} \cdot \underline{k}} \Delta(\underline{i}) \otimes \Delta(\underline{k})\right) \text {. }
$$

Tracing the identity map through the isomorphisms gives the desired $\tau$. Uniqueness follows because $1 \otimes\left(v_{\underline{i}} \otimes v_{\underline{k}}\right)$ generates $\Delta(\underline{i}) \circ \Delta(\underline{k})$.

For a sequence $\underline{\mathbf{i}}$ of elements in $\Delta_{F}$, define

$$
\tau_{k}(\underline{\mathbf{i}}) \in \operatorname{End}(P)
$$

to act by id $\circ \cdots \circ$ id $\circ \tau \circ$ id $\circ \cdots \circ$ id on $\Delta\left(\underline{i}_{1}\right) \circ \cdots \circ \Delta\left(\underline{i}_{n}\right)$, where the $\tau$ is the homomorphism from Lemma 3.10 acting in the $k$-th and $(1+k)$-th place.

For $\alpha \in \Phi$ consider the restriction $R^{\sup (\alpha)}$ of $R$ to the sub-Dynkin diagram where $\alpha$ is supported. From now on we make the following assumption about faces $F$.

Assumption 3.11. For every $\alpha \in \Delta_{F}, R^{\sup (\alpha)}$ satisfies Assumption 1.1 ,

The following is $\mathrm{BKM}$, Theorem 3.3(4)] if $R^{\sup (\alpha)}$ is of finite type, and $\mathrm{M}$, Theorem 18.3] if it is of geometric symmetric affine type over a field of characteristic zero, together with [BKM, Lemma 3.9]. 
Theorem 3.12. For each $\alpha \in \Delta_{F}, \operatorname{End}(\Delta(\alpha)) \cong k\left[x_{\alpha}\right]$, where $x_{\alpha}$ has degree $\alpha \cdot \alpha$, and $\Delta(\alpha)$ is a free $k\left[x_{\alpha}\right]$-module. Furthermore, there is a unique such isomorphism satisfying the following relations in $\operatorname{End}(\Delta(\alpha) \circ \Delta(\alpha)): \tau x_{2}=x_{1} \tau+1$ and $x_{2} \tau=$ $\tau x_{1}+1$, where $x_{1}$ and $x_{2}$ are the endomorphisms $x_{\alpha} \circ$ id and $i d \circ x_{\alpha}$, respectively.

Definition 3.13. $x_{k}(\mathbf{i}) \in \operatorname{End}(P)$ is the element which acts by ido. . oido $x$ oid $\cdots$ oid on $\Delta\left(\underline{i}_{1}\right) \circ \cdots \circ \Delta\left(\underline{i}_{n}\right)$ and by zero on all other summands, where $x$ is the element from Lemma 3.12 acting on $\Delta\left(\underline{i}_{k}\right)$.

Theorem 3.14. Let $\underline{\mathbf{i}}, \mathbf{j} \in \operatorname{Seq}_{F}(\nu)$. Then

$$
\left\{\tau_{w}(\underline{\mathbf{i}}) x_{1}(\underline{\mathbf{i}})^{a_{1}} \cdots x_{n}(\underline{\mathbf{i}})^{a_{n}} \mid w \in \underline{\mathbf{j}} S_{\underline{\mathbf{i}}}, a_{1}, \ldots, a_{n} \in \mathbb{N}\right\}
$$

is a basis of $\operatorname{Hom}(\Delta(\underline{\mathbf{j}}), \Delta(\underline{\mathbf{i}}))$.

Proof. By Theorem 3.12 each $\Delta\left(\underline{i}_{k}\right)$ is a free module for $k\left[x_{k}\right]$. Using this and Lemma 3.10, the endomorphisms in the statement produce linearly independent vectors when applied to the element $v_{\underline{i}_{1}} \otimes \cdots \otimes v_{\underline{i}_{n}}$, where as above $v_{\underline{i}}$ is a chosen vector in $\Delta(\underline{i})$ of minimal degree. Hence they are linearly independent.

By adjunction and Lemma 3.9 .

$$
\begin{aligned}
\operatorname{Hom}(\Delta(\underline{\mathbf{j}}), \Delta(\underline{\mathbf{i}})) & \cong \operatorname{Hom}\left(\Delta\left(i_{1}\right) \otimes \cdots \otimes \Delta\left(i_{n}\right), \operatorname{Res}_{\underline{\mathbf{j}}} \Delta(\underline{\mathbf{i}})\right) \\
& \cong \bigoplus_{w \in \underline{\mathbf{j}} S_{\underline{\mathbf{i}}}} q^{\operatorname{deg} \tau_{w}(\mathbf{i})} \operatorname{End}\left(\Delta\left(i_{1}\right) \otimes \cdots \otimes \Delta\left(i_{n}\right)\right) \\
& \cong \bigoplus_{w \in \underline{\mathbf{j}} S_{\underline{\mathbf{i}}}} q^{\operatorname{deg} \tau_{w}(\mathbf{i})} \bigotimes_{i=1}^{n} \operatorname{End}\left(\Delta\left(i_{i}\right)\right) .
\end{aligned}
$$

Therefore

$$
\operatorname{dim}_{q} \operatorname{Hom}(\Delta(\underline{\mathbf{j}}), \Delta(\underline{\mathbf{i}}))=\sum_{w \in \underline{\mathbf{j}} S_{\underline{\mathbf{i}}}} q^{\operatorname{deg} \tau_{w}(\underline{\mathbf{i}})} \prod_{i=1}^{n}\left(1-q^{\dot{L}_{i} \cdot \dot{L}_{i}}\right)^{-1} .
$$

This dimension count shows that our linearly independent set is a basis.

Corollary 3.15. If $\underline{i}$ and $\underline{k}$ are distinct roots in $\Delta_{F}$, then

$$
\operatorname{End}(\Delta(\underline{i}) \circ \Delta(\underline{k})) \cong k\left[x_{\underline{i}}, x_{\underline{k}}\right] .
$$

3.4. Relations in $\operatorname{End}(P)$. For distinct $\underline{i}, \underline{j} \in \Delta_{F}$, define $Q_{\underline{i j}}(u, v) \in k[u, v]$ by

$$
\tau_{1}(\underline{j i}) \tau_{1}(\underline{i j})=Q_{\underline{i j}}\left(x_{1}(\underline{i j}), x_{2}(\underline{i j})\right) .
$$

These exist by Corollary 3.15] and are homogeneous of degree $-2 \underline{i} \cdot \underline{j}$ by Theorem 3.12. For $\underline{i}=j$, define $Q_{\underline{i i}}=0$. Then $\tau_{1}(\underline{i}) \tau_{1}(\underline{i \underline{i}})=Q_{\underline{i} \underline{i}}\left(x_{1}(\underline{i i}), x_{2}(\underline{i \underline{i}})\right)=0$, since by Theorem 3.14 the degree $-2 \underline{i} \cdot \underline{i}$ Hom space is zero.

Lemma 3.16. If $\underline{i} \neq \underline{j}$, then $x_{1} \tau_{1}(\underline{i j})=\tau_{1}(\underline{i j}) x_{2}$ and $x_{2} \tau_{1}(\underline{i j})=\tau_{1}(\underline{i j}) x_{1}$.

Proof. The module $\Delta(j)$ is generated by $v_{j}$ so there exists $a \in R$ such that $x v_{j}=$ $a v_{j}$. We now compute

$$
\begin{aligned}
\left(x_{1} \tau_{1}-\tau_{1} x_{2}\right)\left(v_{\underline{i}} \otimes v_{\dot{j}}\right) & =x_{1} \psi_{w[\operatorname{ht}(\underline{j}), \operatorname{ht}(\underline{i})]} v_{\dot{1}} \otimes v_{\underline{i}}-\tau_{1}(1 \otimes a) v_{\underline{i}} \otimes v_{\dot{1}} \\
& =\psi_{w[\operatorname{ht}(\underline{j}), \operatorname{ht}(\underline{i})]} x_{1} v_{\dot{1}} \otimes v_{\underline{i}}-(1 \otimes a) \tau_{1} v_{\underline{i}} \otimes v_{\dot{1}} \\
& =\left(\psi_{w[\operatorname{ht}(\underline{j}), \operatorname{ht}(\underline{i})]}(a \otimes 1)-(1 \otimes a) \psi_{w[\operatorname{ht}(\underline{j}), \operatorname{ht}(\underline{i})]}\right) v_{\dot{j}} \otimes v_{\underline{i}} .
\end{aligned}
$$


Apply the straightening relations in the KLR algebra to put $\psi_{w[\operatorname{ht}(j), \operatorname{ht}(\underline{i})]}(a \otimes 1)-$ $(1 \otimes a) \psi_{w[\mathrm{ht}(j), \mathrm{ht}(\underline{i})]}$ in the standard form as a sum of elements of the form $\psi_{w} P$ where $w$ is a permutation and $P$ is a polynomial in the $y_{i}$. Each term that appears has $w$ not greater than or equal to $w[\mathrm{ht}(j), \mathrm{ht}(\underline{i})]$ in Bruhat order.

On the other hand $x_{1} \tau_{1}-\tau_{1} x_{2}$ is a module homomorphism, so Theorem 3.14 shows that

$$
x_{1} \tau_{1}-\tau_{1} x_{2}=\psi_{w[\operatorname{ht}(\underline{j}), \operatorname{ht}(\underline{i})]}\left(A x_{1}+B x_{2}\right)\left(v_{\dot{1}} \otimes v_{\underline{i}}\right)
$$

for some $A, B \in k$, giving a contradiction unless $A=B=0$.

Lemma 3.17. $Q_{\underline{i j}}(u, v)=Q_{\underline{j i}}(v, u)$.

Proof. By the definition of $Q_{\underline{i} \underline{1}}$,

$$
\tau_{1}(\underline{i j}) \tau_{1}(\underline{i i}) \tau_{1}(\underline{i j})=\tau_{1}(\underline{i j}) Q_{\underline{i j}}\left(x_{1}, x_{2}\right) .
$$

On the other hand, using the definition of $Q_{\underline{j i}}$ and the relation in Lemma 3.16 .

$$
\begin{aligned}
\tau_{1}(\underline{i j}) \tau_{1}(\underline{j i}) \tau_{1}(\underline{i j}) & =Q_{\underline{j i}}\left(x_{1}, x_{2}\right) \tau_{1}(\underline{i j}) \\
& =\tau_{1}(\underline{i j}) Q_{\underline{j i}}\left(x_{2}, x_{1}\right) .
\end{aligned}
$$

The result follows by using the basis of $\operatorname{Hom}(\Delta(\underline{i}) \circ \Delta(\underline{j}), \Delta(\dot{j}) \circ \Delta(\underline{i}))$ from Theorem 3.14 .

Lemma 3.18. The following relations hold:

$\tau_{1}(\underline{j k i}) \tau_{2}(\underline{j i k}) \tau_{1}(\underline{i j k})-\tau_{2}(\underline{k i j}) \tau_{1}(\underline{i k j}) \tau_{2}(\underline{i j k})= \begin{cases}\frac{Q_{\underline{i j}}\left(x_{1}, x_{2}\right)-Q_{\underline{i j}}\left(x_{3}, x_{2}\right)}{x_{1}-x_{3}} & \text { if } \underline{i}=\underline{k} \\ 0 & \text { otherwise. }\end{cases}$

Proof. Apply $\tau_{1} \tau_{2} \tau_{1}-\tau_{2} \tau_{1} \tau_{2}$ to $v_{\underline{i}} \otimes v_{j} \otimes v_{\underline{k}}$. By the straightening relations in the KLR algebra, only terms of the form $\bar{T} \psi_{w}\left(v_{\underline{k}} \otimes v_{j} \otimes v_{\underline{i}}\right)$ can appear, where $T$ is a polynomial in the $y_{i}$ and $w$ is a permutation strictly less than $w_{1} w_{2} w_{1}$ in Bruhat order. Here $w_{i}$ is the permutation that acts as $w\left[\mathrm{ht}\left(\underline{i}_{k}\right), \operatorname{ht}\left(\underline{i}_{k+1}\right)\right]$ on the $i, i+1$ factors, and trivially on the other factor.

On the other hand $\tau_{1} \tau_{2} \tau_{1}-\tau_{2} \tau_{1} \tau_{2}$ is a module homomorphism, so Theorem 3.14 severely restricts its possibilities: By the observations of the previous paragraph, this difference must be a composition of at most two $\tau_{i}$ followed by some $x_{i}$. If $\underline{i}$, $\dot{i}$ and $\underline{k}$ are all distinct, this difference must be zero. If $\underline{i}=\dot{i} \neq \underline{k}$, the only terms which perform the right permutation on the factors are of the form $\tau_{1} \tau_{2}$ composed with some $x_{i}$, but these are all of higher degree, so the difference again is zero. The $\underline{i} \neq \hat{j}=\underline{k}$ case is similar. If $\underline{i}=\underline{j}=\underline{k}$, there are no possibilities of the right degree (i.e., $-3 i \cdot i$ ), so the difference is zero.

It remains to consider $\underline{i}=\underline{k} \neq j$. Considerations as above show that $\tau_{1} \tau_{2} \tau_{1}-$ $\tau_{2} \tau_{1} \tau_{2}=P\left(x_{1}, x_{2}, x_{3}\right)$ for some polynomial $P$. Multiplying on the left by $\tau_{1}$ gives

$$
\tau_{1}^{2} \tau_{2} \tau_{1}-\tau_{1} \tau_{2} \tau_{1} \tau_{2}=\tau_{1} P .
$$

Apply the case of this lemma which we have already proven to get

$$
\tau_{1}^{2} \tau_{2} \tau_{1}-\tau_{2} \tau_{1} \tau_{2} \tau_{2}=\tau_{1} P .
$$

Simplifying the $\tau_{i}^{2}$ terms gives

$$
Q_{\underline{j k}}\left(x_{1}, x_{2}\right) \tau_{2} \tau_{1}-\tau_{2} \tau_{1} Q_{\underline{j k}}\left(x_{2}, x_{3}\right)=\tau_{1} P .
$$

Applying the relations from Lemma 3.16 (pushing a dot past a crossing in diagrammatic notation) and using Theorem 3.14, (3.2) uniquely determines $P$. But 
the stated $P$ does satisfy the equation, since this is true in the standard KLR algebra.

Proposition 3.19. $\operatorname{End}(P)$ is a pseudo-KLR algebra.

Remark 3.20. We will prove that $\operatorname{End}(P)$ is actually a KLR algebra in Theorem 3.23 .

Proof. Let $A$ be the pseudo-KLR algebra defined using the root system $\Phi_{F}$ and the polynomials $Q_{i^{\prime} j^{\prime}}(u, v)$ defined in (3.1) for $i^{\prime}, j^{\prime} \in \Delta_{F}$. The above results show that there is a homomorphism from $A$ to $\operatorname{End}(P)$. This is surjective by Theorem 3.14. To show it is an isomorphism, it suffices to show that $\operatorname{dim}_{q} A \leq \operatorname{dim}_{q} \operatorname{End}(P)$. The latter dimension is known by Theorem 3.14. The former dimension is bounded above by this since we can use the usual straightening relations to put each element of $A$ in a standard form.

Lemma 3.21. Let $A$ be a $\mathbb{Z}$-graded algebra with $\operatorname{dim} A_{n}$ finite for all $n$ and zero for sufficiently negative $n$. Let $P$ be a finitely generated projective $A$-module and let $B=\operatorname{End}_{A}(P)$. Then the number of irreducible modules for $B$ is equal to the number of irreducible quotients of $P$, where both counts are taken up to grading shift and isomorphism.

Proof. Write $P=\bigoplus_{i} P_{i}^{\oplus d_{i}}$ where the $P_{i}$ are pairwise non-isomorphic indecomposable projectives and the $d_{i}$ are positive integers. Then

$$
\operatorname{End}(P)=\bigoplus_{i, j} \operatorname{Hom}\left(P_{i}^{d_{i}}, P_{j}^{d_{j}}\right)
$$

The radical of $\operatorname{End}(P)$ is generated by the radicals of each $\operatorname{End}\left(P_{i}^{d_{i}}\right)$ as well as all of $\operatorname{Hom}\left(P_{i}, P_{j}\right)$ for $i \neq j$. The maximal semi-simple quotient of $\operatorname{End}(P)$ is $\oplus_{i} \operatorname{End}\left(\mathrm{hd}\left(P_{i}\right)^{\oplus d_{i}}\right)$, which is a direct sum of matrix algebras. Therefore the number of irreducible representations of $\operatorname{End}(P)$ is equal to the number of irreducible quotients of $P$.

Lemma 3.22. For all $\underline{i} \neq j \in \Delta_{F}$, the coefficient of $u^{-a_{\underline{i j}}}$ in $Q_{\underline{i j}}(u, v)$ is non-zero. Here $a_{\underline{i}, j}$ is the entry in the Cartan matrix for $\Phi_{F}$.

Proof. Let $P_{\left(1-a_{\underline{i j}}\right) \underline{i+j}}$ be the component of $P$ of weight $\left(1-a_{\underline{i j}}\right) \underline{i}+\underline{j}$. By Proposition 3.19. End $P_{\left(1-a_{i j}\right) \underline{i}+j}$ is a pseudo-KLR algebra. Suppose instead that the coefficient of $u^{-a_{\underline{i j}}}$ in $Q_{\underline{i j}}(u, v)$ was zero. By Theorem 2.16 and Lemma 3.21, $P_{\left(1-a_{\underline{i j}}\right) \underline{i+j}}$ must have at least $2-a_{\underline{i j}}$ pairwise non-isomorphic irreducible quotients.

Pick a convex order $\prec$ on $\Phi^{+}$such that $\underline{i}$ and $j$ span a compatible face. If the face is of finite type, then by Theorem 2.25 the number of cuspidal modules for this face is the number of elements of the corresponding rank two crystal, which is $1-a_{\underline{i j}}$, so, this is a contradiction.

Since $\Phi$ is either of finite or symmetric affine type, it remains to consider the case where $\underline{i}$ and $j$ span a root system of type $\widehat{\mathfrak{s l}}_{2}$. Then the minimal imaginary root is $\delta=\underline{i}+\underline{j}$, and $P_{3 \underline{i}+j}$ has at least four pairwise non-isomorphic irreducible quotients. Without loss of generality, $\dot{j} \succ \underline{i}$. In terms of the classification in Theorem 2.19, at least two of them are of the form $\mathrm{hd}(L \circ L(2 \underline{i}))$ where $L$ is a cuspidal $R(\delta)$-module.

Since $P_{3 \underline{i}+\underline{j}}$ is projective, there is a non-zero morphism

$$
P_{3 \underline{i}+\underline{j}} \rightarrow L \circ L(2 \underline{i}) \rightarrow L \circ L(\underline{i}) \circ L(\underline{i}) .
$$


The restriction-coinduction adjunction (2.4) gives a corresponding non-zero morphism

$$
\operatorname{Res}_{\underline{i}, \underline{i}, \delta} P_{3 \underline{i}+\underline{j}} \rightarrow L(\underline{i}) \otimes L(\underline{i}) \otimes L .
$$

The same argument as in the proof of Lemma 3.3 shows that $\operatorname{Res}_{\underline{i}, \underline{i}, \delta} P_{3 \underline{i}+\underline{j}}$ is a direct sum of modules of the form

$$
\Delta(\underline{i}) \otimes \Delta(\underline{i}) \otimes(\Delta(\underline{i}) \circ \Delta(j)) \text { or } \Delta(\underline{i}) \otimes \Delta(\underline{i}) \otimes(\Delta(j) \circ \Delta(\underline{i})) .
$$

By the cuspidality of $L$, there is no non-zero morphism from $\Delta(j) \circ \Delta(\underline{i})$ to $L$. Therefore there must be a non-zero morphism from $\Delta(\underline{i}) \circ \Delta(j)$ to $L$.

By Corollary 3.15 the algebra $\operatorname{End}(\Delta(\underline{i}) \circ \Delta(j))$ is isomorphic to $k[x, y]$, which has a unique irreducible quotient, so by Lemma 3.21 $\Delta(\underline{i}) \circ \Delta(j)$ has a unique irreducible quotient. But we have two non-isomorphic modules $L$ which are both quotients of $\Delta(\underline{i}) \circ \Delta(\underline{j})$, so this is a contradiction.

Theorem 3.23. The algebra $\operatorname{End}(P)$ is a KLR algebra for the root system $\Phi_{F}$.

Proof. This is immediate from Proposition 3.19 and Lemma 3.22

\section{FACE FUnCtORS}

4.1. Definition. Fix a face $F$. Let $R_{F}$ be the graded algebra $\operatorname{End}(P)^{\text {op }}$, where $P$ is the projective object in $\mathcal{C}_{F}$ from 33.2 . Theorem 3.23 shows that $R_{F}^{\mathrm{op}}$ is a KLR algebra for the root system $\Phi_{F}$. The existence of the anti-automorphism $\dagger$ (see (2.2) implies that $R_{F}$ is an isomorphic KLR algebra.

Definition 4.1. The face functor is the functor $\mathcal{F}: X \rightarrow P \otimes_{R_{F}} X$ from $R_{F}$-mod to $\mathcal{C}_{F}$.

The image of $\mathcal{F}$ lies in $\mathcal{C}_{F}$ since $\mathcal{F}$ sends a free rank one $R_{F}$-module to $P, \mathcal{F}$ is right exact and $\mathcal{C}_{F}$ is closed under taking quotients.

Remark 4.2. In finite type, this face functor is an example of the functors constructed by Kashiwara and Park $\mathrm{KP}$ in terms of duality data (see $\mathrm{KP}$, Proposition 3.5]). In affine type, we expect that this is also the case, but we do not have a proof.

\subsection{Categorical properties.}

Theorem 4.3. If $\Phi$ is of finite or affine type and $F$ is of finite type, then $\mathcal{F}$ is an equivalence of categories.

Proof. This is immediate from Theorem 3.7 which shows that $P$ is a projective generator.

Lemma 4.4. For any face $F$, the face functor $\mathcal{F}$ is fully faithful.

Proof. We use the criterion that a left adjoint is fully faithful if and only if the unit of adjunction is a natural isomorphism. The functor $\mathcal{F}$ is left adjoint to $\operatorname{Hom}(P,-)$. The unit of this adjunction is an isomorphism on free modules by the construction of the face KLR algebra as an endomorphism algebra. The general case follows by considering a free resolution since tensoring is right exact and the Hom functor is exact since $P$ is projective.

Lemma 4.5. For all $R_{F}$ modules $A$ and $B$ there is a natural isomorphism

$$
\mathcal{F}(A) \circ \mathcal{F}(B) \cong \mathcal{F}(A \circ B) \text {. }
$$


Proof. Write $R_{F}(\lambda)$ for $\operatorname{End}\left(P_{\lambda}\right)^{\mathrm{op}}$. Let $e_{\lambda \mu}^{F}$ be the image of the unit under the inclusion $R_{F}(\lambda) \otimes R_{F}(\mu) \rightarrow R_{F}(\lambda+\mu)$. The two functors $A \otimes B \mapsto \mathcal{F}(A \circ B)$ and $A \otimes B \mapsto \mathcal{F}(A) \circ \mathcal{F}(B)$ from $R_{F}(\lambda) \otimes R_{F}(\mu)$-mod to $R(\lambda+\mu)$-mod are given by tensoring with the bimodules

$$
P_{\lambda+\mu} \bigotimes_{R_{F}(\lambda+\mu)} R_{F}(\lambda+\mu) e_{\lambda \mu}^{F}
$$

and

$$
R(\lambda+\mu) e_{\lambda \mu} \bigotimes_{R(\lambda) \otimes R(\mu)}\left(P_{\lambda} \otimes P_{\mu}\right)
$$

respectively. These are both canonically isomorphic to the direct sum over all $\underline{i}_{1}, \ldots, \underline{i}_{n} \in \Delta_{F}$ with $\underline{i}_{1}+\cdots+\underline{i}_{l}=\lambda$ and $\underline{i}_{l+1}+\cdots+\underline{i}_{n}=\mu$ of

$$
\Delta\left(\underline{i}_{1}\right) \circ \cdots \circ \Delta\left(\underline{i}_{n}\right) \text {. }
$$

Therefore the functors are equivalent.

4.3. Compatibility with standard modules. Results in this section hold under Assumption 1.1, which gives us access to the theory of standard modules introduced in $[\mathrm{BKM}]$ and $[\mathrm{M}]$. These depend on the convex order $\prec$ and are built out of root modules. The root modules corresponding to real roots have already been introduced, these are the modules $\Delta(\alpha)$. For the indivisible imaginary root $\delta$, we will call the modules denoted $\Delta(\omega)$ in [M] root modules. These are the projective modules in the category of cuspidal $R(\delta)$-modules.

Standard modules are naturally indexed by root partitions. A root partition is a sequence $\lambda=\left(\alpha_{1}^{n_{1}}, \cdots, \alpha_{l}^{n_{l}}\right)$ where $\alpha_{1} \succ \cdots \succ \alpha_{l}$ are indivisible roots, each $n_{i}$ is a positive integer unless $\alpha_{i}=\delta$, in which case it is a collection of partitions. To each term $\alpha_{i}^{n_{i}}$ a standard module $\Delta\left(\alpha_{i}\right)^{\left(n_{i}\right)}$ is constructed. If $\alpha_{i}$ is real, then $\Delta\left(\alpha_{i}\right)^{\text {on }} n_{i}$ is a direct sum of $n_{i}$ ! copies of the module $\Delta\left(\alpha_{i}\right)^{\left(n_{i}\right)}$ with grading shifts. If $\alpha_{i}$ is imaginary, then $\Delta\left(\alpha_{i}\right)^{\left(n_{i}\right)}$ is a summand of a product of certain modules $\Delta(\omega)$ of weight $\delta$ in $\mathcal{C}_{F}$; see $[\mathrm{M}]$ for the details (where this module is denoted $\Delta(\underline{\lambda})$ ). The standard module is then defined to be the indecomposable module

$$
\Delta(\lambda)=\Delta\left(\alpha_{1}\right)^{\left(n_{1}\right)} \circ \cdots \circ \Delta\left(\alpha_{l}\right)^{\left(n_{l}\right)} .
$$

In $[\mathrm{BKM}]$ and $[\mathrm{M}]$ homological properties of these modules are developed which justify the name standard in the setting of affine quasi-hereditary algebras.

Definition 4.6. Fix a convex order $\prec$ and a root $\alpha$. A minimal pair for $\alpha$ is an ordered pair of roots $(\beta, \gamma)$ such that $\alpha=\beta+\gamma, \gamma \prec \beta$, and there is no pair of $\operatorname{roots}\left(\beta^{\prime}, \gamma^{\prime}\right)$ satisfying $\alpha=\beta^{\prime}+\gamma^{\prime}$ and $\gamma \prec \gamma^{\prime} \prec \beta^{\prime} \prec \beta$.

Lemma 4.7. Fix a convex order $\prec$. Let $\Delta$ be a root module with $\operatorname{wt}(\Delta)=\alpha$ and let $(\beta, \gamma)$ be a minimal pair for $\alpha$. Then there exist root modules $\Delta_{\beta}$ and $\Delta_{\gamma}$ with $\operatorname{wt}\left(\Delta_{\beta}\right)=\beta$ and $\operatorname{wt}\left(\Delta_{\gamma}\right)=\gamma$ such that there is a short exact sequence

$$
0 \rightarrow q^{-\beta \cdot \gamma} \Delta_{\beta} \circ \Delta_{\gamma} \stackrel{f_{\beta \gamma}}{\longrightarrow} \Delta_{\gamma} \circ \Delta_{\beta} \rightarrow \Delta^{\oplus m} \rightarrow 0
$$

for some non-zero $m \in \mathbb{N}\left[q, q^{-1}\right]$. Furthermore the homomorphism $f_{\beta \gamma}$ spans the degree zero part of $\operatorname{Hom}\left(q^{-\beta \cdot \gamma} \Delta_{\beta} \circ \Delta_{\gamma}, \Delta_{\gamma} \circ \Delta_{\beta}\right)$.

Conversely suppose that $\Delta_{\beta}$ and $\Delta_{\gamma}$ are root modules with $\operatorname{wt}\left(\Delta_{\beta}\right)=\beta$ and $\operatorname{wt}\left(\Delta_{\gamma}\right)=\gamma$. Then the degree zero part of $\operatorname{Hom}\left(q^{-\beta \cdot \gamma} \Delta_{\beta} \circ \Delta_{\gamma}, \Delta_{\gamma} \circ \Delta_{\beta}\right)$ is one dimensional. If $f$ is any non-zero degree zero homomorphism, then $f$ is injective and coker $f$ is a direct sum of root modules. 
Remark 4.8. The notation, e.g., $\Delta_{\beta}$, as opposed to $\Delta(\beta)$, is because, if $\beta=\delta$, then $\Delta_{\delta}$ can be any of the modules $\Delta(\omega)$ of weight $\delta$.

Proof. In finite type, this is [BKM], Theorem 4.10], and in symmetric affine type it is [M, Lemma 16.1] for real roots and [M, Theorem 17.1] for imaginary roots. The statement about the space of degree zero homomorphisms being one dimensional is not explicitly mentioned, but is clear from the proofs.

Given a face $F$ and a convex order $\prec$ compatible with $F$, we naturally get a convex order $\prec_{F}$ on the face root system $\Phi_{F}$. Thus we can talk about root modules for both $R$ and $R_{F}$.

Lemma 4.9. The face functor $\mathcal{F}$ sends root modules to root modules.

Proof. Let $\Delta$ be a root module for $R_{F}$ and let $\alpha=\operatorname{wt}(\Delta)$. Proceed by induction on the height of $\alpha$, the case $\operatorname{wt}(\Delta) \in \Delta_{F}$ being trivial. If $\alpha \notin \Delta_{F}$ there is a minimal pair $(\beta, \gamma)$ for $\alpha$, and by Lemma 4.7 there is a short exact sequence

$$
0 \rightarrow q^{-\beta \cdot \gamma} \Delta_{\beta} \circ \Delta_{\gamma} \rightarrow \Delta_{\gamma} \circ \Delta_{\beta} \rightarrow \Delta^{\oplus m} \rightarrow 0
$$

where $\Delta_{\beta}$ and $\Delta_{\gamma}$ are root modules with $\operatorname{wt}\left(\Delta_{\beta}\right)=\beta$ and $\operatorname{wt}\left(\Delta_{\gamma}\right)=\gamma$.

By Lemma 4.5 and the fact that $\mathcal{F}$ is right exact, the following is exact:

$$
q^{-\beta \cdot \gamma} \mathcal{F}\left(\Delta_{\beta}\right) \circ \mathcal{F}\left(\Delta_{\gamma}\right) \rightarrow \mathcal{F}\left(\Delta_{\gamma}\right) \circ \mathcal{F}\left(\Delta_{\beta}\right) \rightarrow \mathcal{F}(\Delta)^{\oplus m} \rightarrow 0 .
$$

By the inductive hypothesis, $\mathcal{F}\left(\Delta_{\beta}\right)$ and $\mathcal{F}\left(\Delta_{\gamma}\right)$ are root modules. Since $(\beta, \gamma)$ is a minimal pair, Lemma 4.7 implies that $\mathcal{F}(\Delta)^{\oplus m}$ is a direct sum of root modules. Since root modules are indecomposable, the Krull-Schmidt theorem implies that $\mathcal{F}(\Delta)$ is a root module, as required.

Proposition 4.10. The face functor $\mathcal{F}$ takes standard modules to standard modules.

Proof. Standard modules are built from root modules from a process of inducing and taking direct summands. By Lemma 4.9, $\mathcal{F}$ takes root modules to root modules. The functor $\mathcal{F}$ commutes with induction by Lemma 4.5. Since $\mathcal{F}$ is fully faithful it takes indecomposables to indecomposables, and clearly commutes with taking direct sums.

\subsection{Compatibility with nesting.}

Proposition 4.11. Let $E \subset F$ be nested faces, both satisfying Assumption 3.11. Assume further that $E$ satisfies Assumption 3.11 with respect to the KLR algebra $R_{F}$. Then $\left(R_{F}\right)_{E}$ is isomorphic to $R_{E}$. Furthermore, $\mathcal{F}_{E}$ and $\mathcal{F}_{F} \circ \mathcal{F}_{E}^{F}$ are naturally isomorphic, where $\mathcal{F}_{E}^{F}: R_{E}$-mod $\rightarrow R_{F}$-mod is the face functor for $E$ considered as a face of $\Phi_{F}$.

Proof. Let $P_{F}, P_{E}$ and $P_{E}^{F}$ be the modules used to define the functors $\mathcal{F}_{F}$ and $\mathcal{F}_{E}$ and $\mathcal{F}_{E}^{F}$, respectively. Then

$$
P_{E}=\bigoplus_{\mathbf{i} \in \operatorname{Seq}_{E}} \Delta\left(i_{1}\right) \circ \cdots \circ \Delta\left(i_{n}\right) \text { and } P_{E}^{F}=\bigoplus_{\mathbf{i} \in \operatorname{Seq}_{E}} \Delta_{F}\left(i_{1}\right) \circ \cdots \circ \Delta_{F}\left(i_{n}\right),
$$

where $\Delta(\alpha)$ and $\Delta_{F}(\alpha)$ refer to the root modules for the KLR algebras $R$ and $R_{F}$, respectively. For any $i \in \Delta_{E}, R^{\sup (i)}$ satisfies Assumption 1.1, so, by Lemma 4.9, $\mathcal{F}_{F}\left(\Delta_{F}(i)\right)=\Delta(i)$. By Lemma 4.5 we see $\mathcal{F}_{F}\left(P_{E}^{F}\right)=P_{E}$, so by Lemma 4.4 $\operatorname{End}\left(P_{E}^{F}\right) \cong \operatorname{End}\left(P_{E}\right)$, and hence $\left(R_{F}\right)_{E} \simeq R_{E}$ by definition. 
Since $\mathcal{F}_{F}\left(P_{E}^{F}\right)=P_{E}$ and $R_{E} \cong \operatorname{End}\left(P_{E}\right)^{\text {op }} \cong \operatorname{End}\left(P_{E}^{F}\right)^{\text {op }}$, we obtain an isomorphism of $\left(R, R_{E}\right)$-bimodules

$$
P_{E}^{F} \otimes_{R_{F}} P_{F} \cong P_{E}
$$

This completes the proof.

4.5. Compatibility with crystal operators. In this section we work under Assumption 1.1. Recall $\underline{\mathcal{B}}_{F}$ from Definition 2.22, Let $\mathcal{B}_{F}$ be the set of self-dual simple modules for the KLR algebra $R_{F}$. Recall also the crystal operators on $\underline{\mathcal{B}}_{F}$ and $\mathcal{B}_{F}$ from 92.4 . Theorem 4.3 immediately implies that, in finite type, the face functor gives a bijection $\mathcal{B}_{F} \mapsto \underline{\mathcal{B}}_{F}$, and this intertwines the crystal operators for $\mathcal{B}_{F}$ and the face crystal operators on $\underline{\mathcal{B}}_{F}$. We now prove a weaker version of this that holds in affine type (see Corollary 4.15).

Lemma 4.12. If $L$ is simple, then $\mathcal{F}(L)$ has simple head.

Proof. Every simple module $L$ is the head of a standard module $\Delta$. As $\mathcal{F}$ is right exact, $\mathcal{F}(L)$ is a quotient of $\mathcal{F}(\Delta)$. But $\mathcal{F}(\Delta)$ is standard by Proposition 4.10, so has a simple head. By Lemma $4.4, \mathcal{F}(L) \neq 0$, so this completes the proof.

Proposition 4.13. Let $\Delta_{F}^{\prime}$ be the set of simple roots of $\Phi_{F}$ thought of as its own root system, not as a sub-root system of $\Delta$. For all simple modules $L$ of $R_{F}$ and $i^{\prime} \in \Delta_{F}^{\prime}$,

$$
\operatorname{hd}\left(\mathcal{F}\left(\operatorname{hd}\left(L \circ L\left(i^{\prime}\right)\right)\right)\right) \cong \operatorname{hd}\left(\operatorname{hd} \mathcal{F}(L) \circ \mathcal{F}\left(L\left(i^{\prime}\right)\right)\right),
$$

up to a grading shift, and this is a non-zero simple module.

Remark 4.14. We expect that the grading shift in Proposition 4.13 is unnecessary.

Proof. Since $\mathcal{F}$ is right exact, $\mathcal{F}\left(L \circ L\left(i^{\prime}\right)\right)$ surjects onto $\mathcal{F}\left(\mathrm{hd}\left(L \circ L\left(i^{\prime}\right)\right)\right)$ and hence onto $\operatorname{hd}\left(\mathcal{F}\left(\operatorname{hd}\left(L \circ L\left(i^{\prime}\right)\right)\right)\right)$. This is simple by Lemma 4.12, since by [KL, Lemma 3.9] hd $\left(L \circ L\left(i^{\prime}\right)\right)$ is simple.

On the other hand, by Lemma 4.5 we have $\mathcal{F}\left(L \circ L\left(i^{\prime}\right)\right) \cong \mathcal{F}(L) \circ \mathcal{F}\left(L\left(i^{\prime}\right)\right)$. This surjects onto hd $\mathcal{F}(L) \circ \mathcal{F}\left(L\left(i^{\prime}\right)\right)$ and hence onto hd(hd $\left.\mathcal{F}(L) \circ \mathcal{F}\left(L\left(i^{\prime}\right)\right)\right)$. This last is simple by [TW, Proposition 3.22] since $\mathcal{F}$ sends $L\left(i^{\prime}\right)$ to a simple cuspidal module. Thus both sides of the equation are simple quotients of $\mathcal{F}\left(L \circ L\left(i^{\prime}\right)\right)$.

Choose a convex order on $\Phi_{F}$ with $i^{\prime}$ minimal, and a compatible convex order on $\Phi$. The module $L \circ L\left(i^{\prime}\right)$ is a quotient of $\Delta(L) \circ \Delta\left(i^{\prime}\right)$, and it is immediate from the definition of standard modules (see [M, §24]) that this last is isomorphic to a direct sum of copies of $\Delta\left(f_{i^{\prime}}(L)\right)$. Here $\Delta(L), \Delta\left(f_{i^{\prime}}(L)\right)$ and $\Delta\left(i^{\prime}\right)$ are the standard modules corresponding to $L, f_{i^{\prime}}(L)$ and $L\left(i^{\prime}\right)$. Since $\mathcal{F}$ sends standard modules to standard modules, we see that $\mathcal{F}\left(L \circ L\left(i^{\prime}\right)\right)$ can only have one isomorphism class of simple quotient up to a grading shift, which completes the proof.

Corollary 4.15. The injection $\mathcal{B}_{F} \rightarrow \underline{\mathcal{B}}_{F}$ defined by $L \mapsto$ hd $\mathcal{F}(L)$ intertwines the crystal operators for $\mathcal{B}_{F}$ and the face crystal operators on $\underline{\mathcal{B}}_{F}$ from [TW].

Remark 4.16. If $F$ is of finite type, then, by $[\mathrm{KP}$, Theorem 4.4(ii)(a)], $\mathcal{F}$ takes a simple module either to a simple module or zero (their construction is more general, and by Lemma 4.4 the latter is not possible in our case). This is not true in affine type, as we discuss in 5.4 . Thus taking the head is necessary in Corollary 4.15. 
4.6. Example. Let $\Phi$ be a root system of type $A_{2}^{(1)}$ with simple roots $\alpha_{0}, \alpha_{1}$ and $\alpha_{2}$. Fix the polynomials defining the KLR algebra to be $Q_{i, i+1}(u, v)=s_{i} u+t_{i} v$ where all indices are read modulo 3 . Let $\pi$ be the standard projection from affine roots to finite type roots which sends $\delta$ to zero. Consider

$$
\begin{aligned}
F^{+} & =\pi^{-1}\left\{\alpha_{2}, \alpha_{1}+\alpha_{2}\right\}, \\
F & =\pi^{-1}\left\{\alpha_{1},-\alpha_{1}, 0\right\}, \\
F^{-} & =\pi^{-1}\left\{-\alpha_{2},-\alpha_{1}-\alpha_{2}\right\} .
\end{aligned}
$$

Then $\left(F^{+}, F, F^{-}\right)$is a face according to Definition 2.1, Notice that $F=\{m \delta, m \delta \pm$ $\left.\alpha_{1}\right\}$, so $\Phi_{F}$ is of type $\widehat{\mathfrak{s l}}_{2}$ with simple roots $\beta=\alpha_{1}$ and $\gamma=\alpha_{0}+\alpha_{2}$. The support of both these roots is finite type, so Assumption 3.11 holds, regardless of where the original KLR algebra was of geometric type, and the face functor is defined.

The cuspidal $R(\gamma)$-module has character [02]. Let $v_{\gamma}$ be a lowest degree element of $\Delta(\gamma)$. The endomorphism $x_{\gamma}$ of $\Delta(\gamma)$ (normalized as in Theorem 3.12) satisfies

$$
x_{\gamma} v_{\gamma}=-s_{2}^{-1} y_{1} v_{\gamma}=t_{2}^{-1} y_{2} v_{\gamma} .
$$

Explicit computation shows that

$$
Q_{\beta \gamma}(u, v)=s_{1} t_{0} u^{2}+\left(t_{0} t_{1} t_{2}-s_{0} s_{1} s_{2}\right) u v-s_{0} s_{2} t_{1} t_{2} v^{2} .
$$

Note that

- The coefficient of $u v$ is zero if and only if $s_{0} s_{1} s_{2}=t_{0} t_{1} t_{2}$.

- The discriminant of $Q$ is zero if and only if $s_{0} s_{1} s_{2}+t_{0} t_{1} t_{2}=0$.

These observations imply that

- Examples 3.3 and 3.4 of $[\mathrm{K}]$ are related by a face functor.

- By Lemma 2.14 $R$ is of geometric type if and only if $R_{F}$ is.

\section{IMAGINARY CUSPIDAL REPRESENTATIONS AND AFFINE FACES}

For this section $\Phi$ is of symmetric affine type and, unless otherwise stated, the KLR algebra $R$ is of geometric type. Fix a convex order $\prec$ on $\Phi^{+}$. Let $\delta$ be the minimal imaginary root. We study the category of cuspidal $R(\delta)$-modules. In particular, we show that the endomorphism algebra of a projective generator of the category of cuspidal $R(\delta)$-modules is isomorphic to $k[z] \otimes Z$ where $Z$ is the zigzag algebra from $[\mathrm{HK}]$ corresponding to the underlying finite type Dynkin diagram. We show this by using face functors to reduce to the $\widehat{\mathfrak{s l}}_{3}$ case. We also show that, if $R$ is of geometric type, so is any face KLR algebra $R_{F}$ (see Theorem 5.10).

Let $S(\delta)$ be the quotient of $R(\delta)$ by the two-sided ideal generated by all $e_{\mathbf{i}}$ where $\mathbf{i}$ has a proper prefix $\mathbf{i}^{\prime}$ with $\operatorname{wt}\left(\mathbf{i}^{\prime}\right)$ a sum of roots $\succ \delta$. Then, as discussed in 92.3, the category of $S(\delta)$-modules is equivalent to the category of cuspidal $R(\delta)$ modules, so this agrees with the algebra $S(\delta)$ from [M, $\S 12]$. By [M] $\S 17]$, the simple $S(\delta)$-modules are naturally parametrized by a set $\Omega$ of chamber coweights for an underlying finite type Cartan matrix. For each $\omega \in \Omega$, let $L(\omega)$ be the selfdual irreducible module parametrized by $\omega$ and let $\Delta(\omega)$ be its projective cover in the category of cuspidal $R(\delta)$-modules. Additionally, to each $\omega \in \Omega$, consider the positive real roots $\left(\omega_{-}, \omega_{+}\right)$, defined in [M, $\S 12$ ] (see also [TW, 33.4 ], where $\omega_{-}$is called $\left.\beta_{0}\right)$ which have the following properties:

$$
\begin{aligned}
& \text { - } \omega_{-} \prec \delta \text { and } \omega_{+} \succ \delta \text {, } \\
& \text { - } L(\omega)=\operatorname{hd}\left(L\left(w_{-}\right) \circ L\left(w_{+}\right)\right) \text {, and }
\end{aligned}
$$


- $\{\pi(\beta): \beta \succ \delta\}$ is a positive system for the underlying finite type root system, and $\left\{\pi\left(\omega_{+}\right): \omega \in \Omega\right\}$ is the corresponding set of simple roots. Here $\pi$ is the standard projection from $\$ 4.6$.

For each positive real root $\alpha$ let $E_{\alpha}$ and $E_{\alpha}^{*}$ be the PBW and dual PBW basis vectors for the PBW basis associated to $\prec$, as defined in [M, $\S 9$ ] (see also [BCP, BN]). When the Grothendieck group of $R$-modules is identified with $U_{q}^{+}(\mathfrak{g})$ we have [M], Theorems 9.1 and 18.2] which say that $[L(\alpha)]=E_{\alpha}^{*}$ and $[\Delta(\alpha)]=E_{\alpha}$.

5.1. The Cartan matrix for $S(\delta)$. The following is immediate from [TW, Proposition 3.31 and Lemma 3.44], but for completeness we provide an alternative proof.

Lemma 5.1. For all $x \neq \omega$ in $\Omega$, we have $L(x) \circ L\left(\omega_{-}\right) \cong L\left(\omega_{-}\right) \circ L(x)$.

Proof. By Theorem 2.19 there is a short exact sequence

$$
0 \rightarrow X \rightarrow L(x) \circ L\left(\omega_{-}\right) \rightarrow L\left(x, \omega_{-}\right) \rightarrow 0,
$$

where $L\left(x, \omega_{-}\right)$is the irreducible module associated to the root partition $\left(x, \omega_{-}\right)$ and, by $[\mathrm{M}$, Theorem 10.1(3)], $X$ is a successive extension of grading shifts of modules whose cuspidal decomposition only involves roots between $\omega_{-}$and $\delta$. Since $\pi\left(\omega_{-}\right)$is a negative simple root for the positive system $\pi\left(F^{+}\right)$, there is no way to write $\delta+\omega_{-}$as a non-trivial sum of roots $\prec \delta$, so $X$ is in fact a successive extension of grading shifts of $L\left(\delta+\omega_{-}\right)$. By [M], Lemma 7.5] and [M, Theorem 9.1], we have $[X] \in q \mathbb{N}[q] E_{\delta+\omega_{-}}^{*}$ in the Grothendieck group of $R$-modules, identified with $U_{q}^{+}(\mathfrak{g})$.

By (2.4),

$$
\operatorname{Hom}\left(L\left(\delta+\omega_{-}\right), L(x) \circ L\left(\omega_{-}\right)\right) \cong \operatorname{Hom}\left(\operatorname{Res}_{\omega_{-}, \delta} L\left(\delta+\omega_{-}\right), L\left(\omega_{-}\right) \otimes L(x)\right) .
$$

The proof of [M, Lemma 21.8] shows that $\operatorname{Res}_{\omega_{-}, \delta} L\left(\delta+\omega_{-}\right)$has $L\left(\omega_{-}\right) \otimes L(x)$ appearing with graded multiplicity 1 or 0 . Therefore the only possible homomorphisms between $L\left(\delta+\omega_{-}\right)$and $L(x) \circ L\left(\omega_{-}\right)$are in degree zero. Combined with $[X] \in q \mathbb{N}[q] E_{\delta+\omega_{-}}^{*}$, the only option is $X=0$.

Therefore $L(x) \circ L\left(\omega_{-}\right) \cong L\left(x, \omega_{-}\right)$. Taking duals gives $L\left(\omega_{-}\right) \circ L(x) \cong L\left(x, \omega_{-}\right)$, and the lemma follows.

Lemma 5.2 ([M, Lemma 21.7]). For any $\omega \in \Omega$ there is a short exact sequence

$$
0 \rightarrow q L\left(\delta+\omega_{-}\right) \rightarrow L(\omega) \circ L\left(\omega_{-}\right) \rightarrow L\left(\omega, \omega_{-}\right) \rightarrow 0 .
$$

By Lemmas 5.2 and 5.1

$$
[L(x)] E_{\omega_{-}}^{*}= \begin{cases}{\left[L\left(\omega, \omega_{-}\right)\right]+q E_{\delta+\omega_{-}}^{*}} & \text { if } x=\omega, \\ E_{\omega_{-}}^{*}[L(x)] & \text { otherwise. }\end{cases}
$$

In the first case, apply the bar involution and use (2.5) to obtain

$$
E_{\omega_{-}}^{*}[L(\omega)]=\left[L\left(\omega, \omega_{-}\right)\right]+q^{-1} E_{\delta+\omega_{-}}^{*} .
$$

Thus we have the commutator formula

$$
\left[E_{\omega_{-}}^{*},[L(x)]\right]= \begin{cases}\left(q^{-1}-q\right) E_{\delta+\omega_{-}}^{*} & \text { if } x=\omega, \\ 0 & \text { otherwise. }\end{cases}
$$

Lemma 5.3. Let $x$ and $\omega$ be two chamber coweights. Then

$$
\left[E_{\omega_{-}},[\Delta(x)]\right]= \begin{cases}\left(q+q^{-1}\right) E_{\delta+\omega_{-}} & \text {if } x=\omega, \\ 0 & \text { if } x \text { and } \omega \text { are orthogonal }, \\ E_{\delta+\omega_{-}} & \text {otherwise. }\end{cases}
$$


Proof. The ideas of this proof are in [M, Lemma 21.8]. Indeed, when $x=\omega$, this lemma is the decategorification of the $n=0$ case of [M] Lemma 21.8].

Now suppose that $x \neq \omega$. By [M, Lemma 16.1], there is a short exact sequence

$$
0 \rightarrow \Delta(x) \circ \Delta\left(\omega_{-}\right) \rightarrow \Delta\left(\omega_{-}\right) \circ \Delta(x) \rightarrow C \rightarrow 0
$$

where $C$ is a direct sum of $f(q)$ copies of $\Delta\left(\delta+\omega_{-}\right)$for some $f(q) \in \mathbb{N}\left[q, q^{-1}\right]$. Passing to the Grothendieck group, this decategorifies to

$$
\left[E_{\omega_{-}},[\Delta(x)]\right]=f(q) E_{\delta+\omega_{-}} .
$$

When specialized to $q=1, E_{\omega_{-}}$and $E_{\delta+\omega_{-}}$become the root vectors $e_{w_{-}}$and $e_{w_{-}} \otimes t$, respectively in the Lie algebra, and $[\Delta(x)]$ becomes $h_{x} \otimes t$ by [M], Corollary 17.2]. There is

$$
\left[e_{\omega_{-}}, h_{x} \otimes t\right]=\left\langle x, \omega_{-}\right\rangle e_{\omega_{-}} \otimes t .
$$

Since $f(q) \in \mathbb{N}\left[q, q^{-1}\right]$, equations (5.3) and (5.4) complete the proof when $x$ and $\omega$ are orthogonal. In the one remaining case, they imply that $f(q)=q^{n}$ for some $n \in \mathbb{Z}$. Now compute

$$
\begin{aligned}
f(q) & =\left\langle[C],\left[L\left(\delta+\omega_{-}\right)\right]\right\rangle \\
& =\left\langle E_{\omega_{-}} E_{x}-E_{x} E_{\omega_{-}},\left[L\left(\delta+\omega_{-}\right)\right]\right\rangle \\
& =\left\langle E_{\omega_{-}} E_{x},\left[L\left(\delta+\omega_{-}\right)\right]\right\rangle \\
& =\left\langle E_{\omega_{-}} \otimes E_{x},\left[\operatorname{Res}_{\omega_{-}, \delta} L\left(\delta+\omega_{-}\right)\right]\right\rangle,
\end{aligned}
$$

where the third equality holds by the cuspidality of $L\left(\delta+\omega_{-}\right)$. By $[\mathrm{M}$, Lemma 12.3], $\operatorname{Res}_{\omega_{-}, \delta} L\left(\delta+\omega_{-}\right)$is a successive self-extension of grading shifts of simples of the form $L\left(\omega_{-}\right) \otimes L(y)$ for $y \in \Omega$. The above equations imply that $f(q)$ is the multiplicity of $L\left(\omega_{-}\right) \otimes L(x)$ in the Jordan-Holder filtration of $\operatorname{Res}_{\omega_{-}, \delta} L\left(\delta+\omega_{-}\right)$. Since $L\left(\delta+\omega_{-}\right)$ is self-dual and restriction commutes with duality, we obtain $f(q)=f\left(q^{-1}\right)$. Since $f(q)=q^{n}$, this forces $n=0$, completing the proof.

Proposition 5.4. Fix $x, \omega$ in $\Omega$. The multiplicity of $L(x)$ in $\Delta(\omega)$ is

$$
[\Delta(\omega): L(x)]= \begin{cases}\frac{1+q^{2}}{1-q^{2}} & \text { if } x=\omega, \\ 0 & \text { if } x \text { and } \omega \text { are orthogonal, } \\ \frac{q}{1-q^{2}} & \text { otherwise. }\end{cases}
$$

Proof. Write $[\Delta(y)]=\sum_{x} p_{y, x}[L(x)]$. By (5.1),

$$
\left(q^{-1}-q\right) p_{y, \omega} E_{\delta+\omega_{-}}^{*}=\left[E_{\omega_{-}}^{*},[\Delta(y)]\right] .
$$

The formula (5.2) along with $E_{\alpha}^{*}=\left(1-q^{\alpha \cdot \alpha}\right) E_{\alpha}$ completes the proof.

Corollary 5.5. Suppose $x, \omega \in \Omega$. Then

$$
\operatorname{dim}_{q} \operatorname{Hom}(\Delta(\omega), \Delta(x))= \begin{cases}\frac{1+q^{2}}{1-q^{2}} & \text { if } x=\omega, \\ 0 & \text { if } x \text { and } \omega \text { are orthogonal, } \\ \frac{q}{1-q^{2}} & \text { otherwise. }\end{cases}
$$


5.2. Faces in geometric type. The following is immediate from [M, Lemma 15.1].

Lemma 5.6. Assume $R$ has the standard choice of parameters from $[\mathrm{M}]$. Let $S^{\prime}(\delta)$ be the sub-algebra of $S(\delta)$ generated by the $e_{\mathbf{i}}, y_{j}-y_{j+1}$ and $\psi_{k}$. Then $S(\delta) \cong$ $k[z] \otimes S^{\prime}(\delta)$ where $z$ has degree 2 .

Theorem 5.7. Let $\omega$ be a chamber coweight. Then $\operatorname{End}(\Delta(\omega)) \cong k[z, \epsilon] /\left(\epsilon^{2}\right)$ where $z$ and $\epsilon$ are in degree 2 .

Proof. We can assume $R$ has the standard choice of parameters from [M]. Then

$$
\begin{aligned}
\operatorname{End}_{R(\delta)} \Delta(\omega) & \cong \operatorname{End}_{S(\delta)} \Delta(\omega) \\
& \cong k[z] \otimes \operatorname{End}_{S^{\prime}(\delta)}(\Delta(\omega) / z \Delta(\omega)) \\
& \cong k[z] \otimes k[\epsilon] /\left(\epsilon^{2}\right)
\end{aligned}
$$

The second isomorphism holds by Lemma 5.6 and the fact that $\Delta(\omega)$ is a projective $S(\delta)$-module. The third holds by Corollary [5.5.

Lemma 5.8. If $R$ is a geometric KLR algebra of symmetric affine type and $F$ is a face with root system $\Phi_{F}$ of type $\widehat{\mathfrak{s l}}_{2}$, then $R_{F}$ is of geometric type.

Proof. Consider the face functor $\mathcal{F}: R_{F}$-mod $\rightarrow R$-mod. Let $Q_{0^{\prime} 1^{\prime}}(u, v)$ be the quadratic polynomial defining the KLR algebra $R_{F}$. Use the convex order where $1^{\prime} \succ 0^{\prime}$, and let $\Delta$ be the unique indecomposable projective in the category of cuspidal $R_{F}(\delta)$ modules. Direct computation shows $\operatorname{End}(\Delta) \cong k[u, v] / Q_{0^{\prime} 1^{\prime}}(u, v)$ and that there is a short exact sequence

$$
0 \rightarrow q^{2} \Delta\left(1^{\prime}\right) \circ \Delta\left(0^{\prime}\right) \rightarrow \Delta\left(0^{\prime}\right) \circ \Delta\left(1^{\prime}\right) \rightarrow \Delta \rightarrow 0 .
$$

The argument in the proof of Lemma 4.9 then shows that $\mathcal{F}(\Delta)$ is a root module for $R$ so, by Theorem [5.7. $\operatorname{End}(\mathcal{F}(\Delta)) \cong k[z, \epsilon] / \epsilon^{2}$. Since face functors are fully faithful,

$$
k[u, v] / Q_{0^{\prime} 1^{\prime}}(u, v) \cong k[z, \epsilon] / \epsilon^{2},
$$

which implies that $Q_{0^{\prime} 1^{\prime}}(u, v)$ has discriminant zero. Then $R_{F}$ is of geometric type by Lemma 2.14

Any face of type $\widehat{\mathfrak{s l}}_{2}$ faces inside $\widehat{\mathfrak{s l}}_{n}$ satisfies Assumption 3.11, so the face functor is defined, regardless of whether the original KLR algebra was of geometric type.

Lemma 5.9. Let $R$ be a KLR algebra of type $\widehat{\mathfrak{s l}}_{n}$. Let $F$ be the face of type $\widehat{\mathfrak{s l}}_{2}$ defined by

$$
\begin{aligned}
F^{+} & =\pi^{-1}\left\{\Phi_{0}^{+} \backslash\left\{\alpha_{1}\right\}\right\}, \\
F & =\pi^{-1}\left\{\alpha_{1},-\alpha_{1}, 0\right\}, \\
F^{-} & =\pi^{-1}\left\{\Phi_{0}^{-} \backslash\left\{-\alpha_{1}\right\}\right\},
\end{aligned}
$$

where $\Phi_{0}$ is the underlying finite type root system, and $\pi$ is the standard projection sending $\delta$ to 0 . If $R_{F}$ is of geometric type, then $R$ is also of geometric type.

Proof. We can take $I=\mathbb{Z} / n \mathbb{Z}$. Let the polynomials defining $R$ be $Q_{i, i+1}(u, v)=$ $s_{i} u+t_{i} v$ for some $s_{i}, t_{i} \in k^{\times}$.

The simple roots of $\Phi_{F}^{+}$are $\underline{0}=1$ and $\underline{1}=2+\cdots+n$. The corresponding simple cuspidal modules are both one dimensional, with characters [1] and $[n \cdots 32]$, 
respectively. Let $\mathbf{i}=(n, \ldots, 3,2)$. By cuspidality, for each $1<j<n, \psi_{n-j}$ acts by zero on $\Delta(\underline{1})$, so $\Delta(\underline{1})$ is a cyclic module over $k\left[y_{1}, \ldots, y_{n-1}\right]$. Since

$$
\psi_{n-j}^{2} e_{\mathbf{i}}=Q_{j+1, j}\left(y_{n-j}, y_{n-j+1}\right) e_{\mathbf{i}}=\left(t_{j} y_{n-j}+s_{j} y_{n-j+1}\right) e_{\mathbf{i}},
$$

the elements $t_{j} y_{n-j}$ and $-s_{j} y_{n-j+1}$ act the same on $\Delta(\underline{1})$. Since $\Delta(\underline{1})$ is infinite dimensional and finitely generated, some $y_{j}$ must act non-nilpotently, and the above relation implies all do. Thus

$$
\left(t_{2} t_{3} \cdots t_{n-1}\right) y_{1}, \quad(-1)^{n}\left(s_{2} s_{3} \cdots s_{n-1}\right) y_{n-1} \quad \text { and } \quad \lambda x_{1}
$$

act the same on $\Delta(\underline{1})$ for some $\lambda \in k^{\times}$, where $x_{\underline{1}}$ is as in Theorem 3.12 .

By the definitions of $Q_{\underline{0}, \underline{1}}$ and $\tau$ and the fact that $\tau$ is a homomorphism, for any $v \in \Delta(\underline{0})$ and $w \in \Delta(\underline{1})$,

$$
\begin{aligned}
Q_{\underline{0}, \underline{1}}\left(x_{\underline{0}}, x_{\underline{1}}\right)(v \otimes w) & =\tau_{1}(\underline{1}, \underline{0}) \tau_{1}(\underline{0}, \underline{1})(v \otimes w) \\
& =\tau_{1}(\underline{1}, \underline{0})\left(\psi_{1} \psi_{2} \cdots \psi_{n-1}\right)(w \otimes v) \\
& =\left(\psi_{1} \psi_{2} \cdots \psi_{n-1}\right) \tau_{1}(\underline{1}, \underline{0})(w \otimes v) \\
& =\left(\psi_{1} \psi_{2} \cdots \psi_{n-1}\right)\left(\psi_{n-1} \psi_{n-2} \cdots \psi_{1}\right)(v \otimes w) .
\end{aligned}
$$

Each $\psi_{k}$ acts on strands of colors 1 and $n-k+1$, so the KLR algebra relations give

$$
\begin{aligned}
Q_{\underline{0}, \underline{1}}\left(x_{\underline{0}}, x_{\underline{1}}\right)(v \otimes w) & =Q_{1 n}\left(y_{1}, y_{2}\right) Q_{12}\left(y_{1}, y_{n}\right)(v \otimes w) \\
& =\left(t_{n} y_{1}+s_{n} y_{2}\right)\left(s_{1} y_{1}+t_{1} y_{n}\right)(v \otimes w) .
\end{aligned}
$$

By definition $x_{\underline{0}}$ acts on $\Delta(\underline{0})$ as $y_{1}$, and $x_{1}$ acts on $\Delta(\underline{1})$ as in (5.5) (with a shift in index of the $\bar{y}_{i}$ due to the tensor factor $\bar{\Delta}(\underline{0})$ on the left), so, for some $\lambda \in k^{\times}$,

$$
Q_{\underline{0}, \underline{1}}(u, v)=\left(t_{n} u+\frac{\lambda s_{n}}{t_{2} t_{3} \cdots t_{n-1}} v\right)\left(s_{1} u+\frac{\lambda t_{1}}{(-1)^{n} s_{2} s_{3} \cdots s_{n-1}} v\right) .
$$

This has discriminant zero if and only if $s_{1} s_{2} \cdots s_{n}=(-1)^{n} t_{1} t_{2} \cdots t_{n}$. The lemma follows from Lemma 2.14 which characterizes geometric type KLR algebras.

Theorem 5.10. Suppose $\Phi$ is symmetric and of either finite or affine type. Fix a KLR algebra $R$ of geometric type for $\Phi$, and, if $\Phi$ is affine, assume $R$ is defined over a field of characteristic zero. Then, for any face $F$, the KLR algebra $R_{F}$ is also of geometric type.

Proof. Without loss of generality, $F$ is irreducible. The statement is trivial unless $R_{F}$ is of type $\widehat{\mathfrak{s l}}_{n}$ for some $n$, since otherwise by Lemma 2.14 all KLR algebras are geometric. So assume $R_{F}$ is type $\widehat{\mathfrak{s l}}_{n}$. Consider the standard face $E$ of type $\widehat{\mathfrak{s l}}_{2}$ inside $F$ (i.e., the sub-face as defined in Lemma 5.9) and the face functors

$$
R_{E} \text {-mod } \rightarrow R_{F} \text {-mod } \rightarrow R \text {-mod. }
$$

These are well defined since the two simple roots of $E$ both have support in a finite type sub-Dynkin-diagram of $\Phi_{F}$. By Proposition 4.11 this composition of face functors agrees with the one-step face functor, so by Lemma 5.8, $R_{E}$ is of geometric type. But then, by Lemma [5.9, $R_{F}$ is also of geometric type. 
5.3. The zigzag algebra. Let $\Gamma$ be a connected graph (the most important case for us is an ADE Dynkin diagram). The zigzag algebra $Z_{\Gamma}$ associated to $\Gamma$, as defined in $\left[\mathrm{HK}\right.$, is a graded algebra with basis elements $e_{i}$ for each vertex $i, h_{i j}$ for each ordered pair $i, j$ of vertices with an edge from $i$ to $j$, and $w_{i}$ for each vertex $i$. The elements $e_{i}$ are in degree zero, $h_{i j}$ are in degree one and $w_{i}$ are in degree two. Multiplication is given by

$$
\begin{aligned}
e_{i} e_{j} & =\delta_{i j} e_{j}, \\
e_{i} h_{j k} & =\delta_{i j} h_{j k}, \\
h_{i j} e_{k} & =\delta_{j k} h_{i j}, \\
e_{i} w_{j} & =w_{j} e_{i}=\delta_{i j} w_{i}, \\
h_{i j} h_{k l} & =\delta_{j k} \delta_{i l} w_{i},
\end{aligned}
$$

and all other products of basis elements are zero.

Example 5.11. If $\Gamma$ is a single vertex, then $Z_{\Gamma}=\operatorname{span}\{e, w\} \simeq k[w] / w^{2}$, where $w$ has degree two. If $\Gamma$ has no isolated points, then $Z_{\Gamma}$ is generated by the idempotents $e_{i}$ and the degree 1 elements $h_{i j}$.

Lemma 5.12. Let $R$ be a geometric KLR algebra for $\widehat{\mathfrak{s l}}_{3}$. Then $S(\delta)$ is isomorphic to the tensor product of $k[z]$ and the $\mathfrak{s l}_{3}$-zigzag algebra, where $z$ has degree two.

Proof. We can take $I=\mathbb{Z} / 3$, and the defining polynomials for $R$ to be $Q_{i, i+1}(u, v)=$ $u-v$. By applying an outer automorphism of the Dynkin diagram of $\widehat{\mathfrak{s l}}_{3}$ we can assume without loss of generality that the two cuspidal simple modules of $R(\delta)$ have characters [012] and [021] or [120] and [210]. We consider only the first case, since the second follows by a symmetric argument.

Consider $S^{\prime}(\delta)$ from Lemma 5.6. The irreducible representations are one dimensional, so the Cartan matrix computed in Proposition 5.4 tells us its dimension. Specifically $e_{012} S^{\prime}(\delta) e_{012}$ has dimension $1+q^{2}$ and $e_{012} S^{\prime}(\delta) e_{021}$ has dimension $q$. It is now straightforward to see that $\left\{e_{012}, \psi_{2} e_{012},\left(y_{2}-y_{3}\right) e_{012}, e_{021}, \psi_{2} e_{021},\left(y_{2}-\right.\right.$ $\left.\left.y_{3}\right) e_{021}\right\}$ is a basis of $S^{\prime}(\delta)$, and that this is isomorphic to the $A_{2}$ zigzag algebra.

Theorem 5.13. Consider a geometric KLR algebra of symmetric affine type. Let $\delta$ be the smallest imaginary root and fix a convex order $\prec$. The category of cuspidal $R(\delta)$-modules is equivalent to the category of modules over the algebra $k[z] \otimes Z$ where $Z$ is the zigzag algebra corresponding to the underlying finite type Dynkin diagram.

Proof. The direct sum of the modules $\Delta(\omega)$ and their grading shifts is a projective generator of the category of $S(\delta)$-modules, so by Morita theory it suffices to show

$$
\text { End }\left(\bigoplus_{\omega \in \Omega} \Delta(\omega)\right) \cong Z \otimes k[z] \text {. }
$$

For the case of $\widehat{\mathfrak{s l}}_{2}$ this is immediate from Lemma 5.6.

Otherwise, Corollary [5.5] shows the endomorphism algebra has the right dimension. Let $x$ and $y$ be two elements of $\Omega$ connected by an edge. By Corollary 5.5 there is a unique up to scalar non-zero degree 1 morphism $h_{x y}: \Delta(x) \longrightarrow \Delta(y)$. By Theorem 5.7, for all $x \in \Omega$ there is unique up to scalar non-zero degree 2 morphism $\epsilon_{x}: \Delta(x) \longrightarrow \Delta(x)$ which squares to zero. If we can show that $h_{x y} \circ h_{y x}$ is a non-zero multiple of $\epsilon_{x}$, then we can rescale the $h_{x y}$ to ensure that this scalar is one, which will complete the proof. 
Choose a linear function $c$ such that $c\left(x_{+}\right)=c\left(y_{+}\right)=c(\delta)=0$, and $c(\alpha)>0$ for all $\alpha$ such that $\pi(\alpha)$ is another simple root in the positive system $\{\pi(\beta): \beta \succ \delta\}$. The construction in 22.1 gives a convex pre-order such that the span of $x_{ \pm}, y_{ \pm}$ and $\delta$ is a face $F$, and by [TW] Lemma 1.14] this can be refined to a convex total order with the same positive system. By [M, Theorem 13.1 and Theorem 17.3], the category of $S(\delta)$-modules only depends on this system, so without loss of generality we can use the new order.

Consider the face functor

$$
R_{F} \text {-mod } \rightarrow R \text {-mod. }
$$

This is fully faithful and sends standard modules to standard modules, so, to prove that $h_{x y} \circ h_{y x}$ is a non-zero multiple of $\epsilon_{x}$, it suffices to prove this for $R_{F}$. But $R_{F}$ is of type $\widehat{\mathfrak{s l}}_{3}$ and by Theorem 5.10 is of geometric type, so this is immediate from Lemma 5.12 .

Remark 5.14. Kleshchev and Muth $[\mathrm{KM}]$ have independently proven Theorem 5.13 for balanced convex orders. Their methods rely on case-by-case computations which we have avoided. They also discuss analogues for $R(n \delta)$ with $n>1$, where they show that the category of cuspidal modules is equivalent to the category of modules for an "affine zigzag algebra". In CL the affine zigzag algebra (with some minor sign differences) appears in the categorification of the Heisenberg algebra (as $\operatorname{End}_{\mathcal{H}^{\Gamma}}\left(P^{n}\right)$ ). Together, these results could perhaps be interpreted as a type of imaginary face functor, with the Heisenberg algebra playing the role of the KacMoody (or Borcherds) algebra of the face.

5.4. Example. Continue the example from $\$ 4.6$, which considers a face $F$ of type $\widehat{\mathfrak{s l}}_{2}$ inside $\widehat{\mathfrak{s l}}_{3}$. Let $0^{\prime}, 1^{\prime}, \delta^{\prime}$ denote the simple roots and the minimal imaginary root for $\widehat{\mathfrak{s l}}_{2}$. Choose the convex order with $0^{\prime} \prec 1^{\prime}$ and let $\Delta\left(\delta^{\prime}\right)$ be the projective cover of $L\left(\delta^{\prime}\right)$ in the cuspidal category. Then $L\left(\delta^{\prime}\right)$ is one dimensional. There is only one indecomposable projective, so the graded dimension of $\Delta\left(\delta^{\prime}\right)$ agrees with that of $S\left(\delta^{\prime}\right)$, which by Lemma 5.6 is $\frac{1+q^{2}}{1-q^{2}}$. Thus there is an exact sequence

$$
2 q^{2} \Delta\left(\delta^{\prime}\right) \rightarrow \Delta\left(\delta^{\prime}\right) \rightarrow L\left(\delta^{\prime}\right) \rightarrow 0 .
$$

Choose a convex order on $\Phi^{+}$compatible with the $\widehat{\mathfrak{s l}}_{2}$ convex order on $F$. The corresponding chamber coweights are the fundamental coweights $\omega_{1}$ and $\omega_{2}$. Let $\mathcal{F}$ be the face functor associated to $F$ which sends $\Delta\left(0^{\prime}\right)$ to $\Delta\left(\alpha_{2}+\alpha_{0}\right)$, so that $\mathcal{F}\left(\Delta\left(\delta^{\prime}\right)\right)=\Delta\left(\omega_{2}\right)$. Since $\mathcal{F}$ is right exact, Lemma 4.9 implies that (5.6) is sent to an exact sequence

$$
2 q^{2} \Delta\left(\omega_{2}\right) \rightarrow \Delta\left(\omega_{2}\right) \rightarrow \mathcal{F}\left(L\left(\delta^{\prime}\right)\right) \rightarrow 0 .
$$

By Theorem 5.13 (or Lemma 5.12 in this case) the category of cuspidal $R(\delta)$ modules is equivalent to the category of $Z_{\Gamma} \otimes k[z]$ modules. The zigzag algebra $Z_{\Gamma}=\operatorname{span}\left\{e_{1}, e_{2}, h_{12}, h_{21}, w_{1}, w_{2}\right\}$ has two simples $S_{1}, S_{2}$, and the corresponding projective covers are of the form $Z_{\Gamma} e_{1}$ and $Z_{\Gamma} e_{2}$. Then $h_{12} e_{2}$ corresponds to a copy of $q S_{1}$ in $Z_{\Gamma} e_{2}$. Under the equivalence of categories, $Z_{\Gamma} e_{2}$ is sent to $\Delta\left(\omega_{2}\right)$ and $S_{1}$ to $L\left(\omega_{1}\right)$, so we see that $\Delta\left(\omega_{2}\right)$ has a copy of $L\left(\omega_{1}\right)$ in degree 1 . This cannot be in the image of $2 q^{2} \Delta\left(\omega_{2}\right)$, so it survives in $\mathcal{F}\left(L\left(\delta^{\prime}\right)\right)$, and hence $\mathcal{F}\left(L\left(\delta^{\prime}\right)\right)$ is not simple.

Here $\mathcal{F}$ is not an equivalence of categories and Theorem 3.7 does not hold as stated for faces not of finite type. 


\section{ACKNOWLEDGMENTS}

The ideas for this paper arose during the workshop "Algebraic Lie theory and representation theory" at ICMS in Scotland, September 2014. We thank the organizers of that event for a great meeting. We also thank the anonymous referees for many insightful suggestions. The first author was partially supported by ARC grant DE150101415. The second author was partially supported by NSF grant DMS-1265555.

\section{REFERENCES}

[BCP] Jonathan Beck, Vyjayanthi Chari, and Andrew Pressley, An algebraic characterization of the affine canonical basis, Duke Math. J. 99 (1999), no. 3, 455-487, DOI 10.1215/S00127094-99-09915-5. MR:1712630

[BN] Jonathan Beck and Hiraku Nakajima, Crystal bases and two-sided cells of quantum affine algebras, Duke Math. J. 123 (2004), no. 2, 335-402. MR2066942

[BKM] Jonathan Brundan, Alexander Kleshchev, and Peter J. McNamara, Homological properties of finite-type Khovanov-Lauda-Rouquier algebras, Duke Math. J. 163 (2014), no. 7, 1353-1404, DOI 10.1215/00127094-2681278. MR3205728

[CL] Sabin Cautis and Anthony Licata, Heisenberg categorification and Hilbert schemes, Duke Math. J. 161 (2012), no. 13, 2469-2547, DOI 10.1215/00127094-1812726. MR2988902

[HK] Ruth Stella Huerfano and Mikhail Khovanov, A category for the adjoint representation, J. Algebra 246 (2001), no. 2, 514-542, DOI 10.1006/jabr.2001.8962. MR.1872113

[KKKO] Seok-Jin Kang, Masaki Kashiwara, Myungho Kim, and Se-jin Oh, Monoidal categorification of cluster algebras II. arXiv:1505.03241

[K] Masaki Kashiwara, Notes on parameters of quiver Hecke algebras, Proc. Japan Acad. Ser. A Math. Sci. 88 (2012), no. 7, 97-102, DOI 10.3792/pjaa.88.97. MR2946856

[KP] Masaki Kashiwara and Euiyong Park, Affinizations and R-matrices for quiver Hecke algebras. arXiv:1505.03241. To appear in JEMS.

[KM] A. Kleshchev and R. Muth, Affine zigzag algebras and imaginary strata for KLR algebras. arXiv: 1505.03241

[KL] Mikhail Khovanov and Aaron D. Lauda, A diagrammatic approach to categorification of quantum groups. I, Represent. Theory 13 (2009), 309-347, DOI 10.1090/S1088-416509-00346-X. MR2525917

[KL2] Mikhail Khovanov and Aaron D. Lauda, A diagrammatic approach to categorification of quantum groups II, Trans. Amer. Math. Soc. 363 (2011), no. 5, 2685-2700, DOI 10.1090/S0002-9947-2010-05210-9. MR2763732

[LV] Aaron D. Lauda and Monica Vazirani, Crystals from categorified quantum groups, Adv. Math. 228 (2011), no. 2, 803-861, DOI 10.1016/j.aim.2011.06.009. MR2822211

[M] Peter. J. McNamara, Representations of Khovanov-Lauda-Rouquier Algebras III: Symmetric Affine Type. arXiv:1511.05905 To appear in Math Z.

[R] Raphael Rouquier, 2-Kac-Moody algebras. arXiv:1505.03241

[TW] Peter Tingley and Ben Webster, Mirković-Vilonen polytopes and KhovanovLauda-Rouquier algebras, Compos. Math. 152 (2016), no. 8, 1648-1696, DOI 10.1112/S0010437X16007338. MR.3542489

School of Mathematics and Physics, University of Queensland, St Lucia, QLD, AusTRALIA

E-mail address: maths@petermc.net

Department of Mathematics and Statistics, Loyola University, Chicago, Illinois 60660

E-mail address: ptingley@luc.edu 